\title{
A novel self-learning weighted fuzzy local information clustering algorithm integrating local and non-local spatial information for noise image segmentation
}

\author{
Qiuyu Song $^{1}$ (D) . Chengmao $\mathrm{Wu}^{1} \cdot$ Xiaoping Tian $^{1} \cdot$ Yue Song $^{1} \cdot$ Xiaokang Guo $^{1}$
}

Accepted: 26 July 2021 / Published online: 9 September 2021

(C) The Author(s) 2021

\begin{abstract}
Fuzzy clustering algorithm (FCM) can be directly used to segment images, it takes no account of the neighborhood information of the current pixel and does not have a robust segmentation noise suppression. Fuzzy Local Information Cmeans Clustering (FLICM) is a widely used robust segmentation algorithm, which combines spatial information with the membership degree of adjacent pixels. In order to further improve the robustness of FLICM algorithm, non-local information is embedded into FLICM algorithm and a fuzzy C-means clustering algorithm has local and non-local information (FLICMLNLI) is obtained. When calculating distance from pixel to cluster center, FLICMLNLI algorithm considers two distances from current pixel and its neighborhood pixels to cluster center. However, the algorithm gives the same weight to two different distances, which incorrectly magnifies the importance of neighborhood information in calculating the distance, resulting in unsatisfactory image segmentation effects and loss of image details. In order to solve this problem, we raise an improved self-learning weighted fuzzy algorithm, which directly obtains different weights in distance calculation through continuous iterative self-learning, then the distance metric with the weights obtained from self-learning is embedded in the objective function of the fuzzy clustering algorithm in order to improve the segmentation performance and robustness of the algorithm. A large number of experiments on different types of images show that the algorithm can not only suppress the noise but also retain the details in the image, the effect of segmenting complex noise images is better, and it provides better image segmentation results than the existing latest fuzzy clustering algorithms.
\end{abstract}

Keywords Image segmentation $\cdot$ Fuzzy clustering $\cdot$ Distance measurement $\cdot$ Self-learning $\cdot$ Non-local information

\section{Introduction}

With the rapid development of computer technology, digital image technology has spread to industrial inspection, environmental monitoring, military and space exploration and other multidisciplinary fields, making image processing technology attract the attention of many domestic and foreign scholars [1]. Image segmentation [2, 3] is a key link in image processing technology, as well as the key to image understanding and machine vision. It can be used for pre-processing tasks such as scene analysis, image recognition, and object detection. The essence

Qiuyu Song

751556353@qq.com

1 Xi' an University of Posts and Telecommunications, Xi' an 710100, China of image segmentation is the process of segmenting a given image into several disjoint regions, and its accuracy directly affects the subsequent feature extraction and target recognition. Therefore, image segmentation is the key stage of the entire image processing, which has been widely used in image understanding [4], significance detection [5-8], three-dimensional reconstruction [9, 10], high-resolution medical image reconstruction $[11,12]$ and other fields. So far, many image segmentation algorithms have been proposed, such as threshold method [13], graph cutting method [14], spectral clustering algorithm [15], fuzzy clustering algorithm [16] and convolutional neural network algorithm [17]. Due to its fast and effective segmentation characteristics, fuzzy clustering algorithms have been widely used in aerospace remote sensing image processing [18, 19], medical image diagnosis [20, 21], face recognition and fingerprint recognition [22, 23] and many other fields. In the process of image segmentation, there 
are a series of problems such as the loss of information in imaging, the uncertainty of visual perception gray level, and the fuzziness of the image itself. Therefore, the use of fuzzy theory to solve this series of problems in image segmentation has become a research trend. Among them, fuzzy clustering [24] combined with fuzzy theory [25, 26] and clustering technology is a research hotspot. All in all, when extracting and detecting objects in an image, image segmentation technology usually becomes an indispensable step. Therefore, the in-depth study of image segmentation algorithms has important research significance. However, due to many reasons, the image will be disturbed by some noise during the process of acquisition and transmission, and the image itself has problems such as uncertainty and complexity, which greatly reduces the segmentation of the fuzzy clustering algorithm performance. Therefore, it is still of great theoretical significance and research value that how to reduce the noise of noisy images under different noise intensity conditions, and how to restore the detailed information of original images as much as possible while ensuring the robustness of clustering algorithm to noise.

The traditional Fuzzy Mean Clustering(FCM) [27, 28] algorithm proposed by Dunn has been widely used in image segmentation. This algorithm has a good segmentation effect when segmenting images that are not affected by noise. FCM uses Euclidean distance and is not robust. It only considers the difference in grayscale between pixels when classifying, and does not consider neighboring pixels. Therefore, the algorithm is extremely sensitive to noise and outliers, and when segmenting an image with a low signal-to-noise ratio(SNR), the segmentation effect is not good. In recent years, many scholars have introduced spatial information on the basis of the original FCM algorithm, thereby improving the robustness of the FCM algorithm and obtaining better segmentation results. Some of the representative algorithms are as follows: Ahmed et al. [29] added the neighborhood information of the pixel to the objective function of the FCM algorithm and proposed a fuzzy mean clustering with spatial constraints(FCM_s or BCFCM). Although the BCFCM algorithm can achieve better segmentation results, each iteration needs to calculate the neighborhood information of the pixel, which reduces the segmentation efficiency of the BCFCM algorithm. In response to this situation, Chen and Zhang [30] proposed FCM_s1 and FCM_s2. The algorithm replaces the neighborhood item with the neighborhood mean or median, which greatly reduces the calculation time. Szilagyi et al. [31] proposed an enhanced FCM(EnFCM), which generates filtered images in advance from the original image and its mean value in its neighborhood, and clustering is performed based on its gray histogram. Each of the above algorithms introduces a spatial parameter, which is manually specified according to the situation. However, it is difficult to determine the optimal parameters because the parameters depend heavily on noise, but the type and intensity of noise are unknown in advance. For this reason, Cai et al. [32] combined local spatial information and pixel gray features, introduced new factors to obtain new linear weighted sum images, and proposed a fast-generalized fuzzy C-means clustering algorithm(FGFCM). In addition, Krinidis and Chatzis [33] proposed fuzzy local information mean clustering(FLICM) with a certain adaptive ability, which integrates spatial information to construct local fuzzy factors to ensure noise sensitivity and preservation of details.

Up to now, there are still many scholars devoted to the study of how to better achieve image segmentation. Haiping Yu et al. [34] proposed a new region-based active contour model. It improves the inaccuracy of segmentation in the local-based model, which only considers the rough local information but does not consider the spatial relationship between the center pixel and its neighborhood. To further accurately segment medical images, they proposed a novel edge-based active contour model (ACM) for medical image segmentation [35]. In addition, in order to improve the image filtering by Gaussian filter, resulting in the loss of image edge gradient information, they also proposed a local region model based on adaptive bilateral filter for noise image segmentation [36]. Recently, Zhang Xiaofeng et al. [37] improved the FLICM algorithm and proposed an algorithm with local and non-local information(FLICMLNLI). The algorithm makes full use of non-local information with the help of self-similarity, while retaining the original information of the picture through back-projection, thereby improving the robustness of the algorithm. The problem with this algorithm is that when the current pixel information and the local information of the pixel appear in the formula for calculating the Euclidean distance, the two have the same weight. This calculation method erroneously magnifies the role of the local information of the pixel in calculating the distance. The problem caused by too much neighborhood information is that the denoising ability is enhanced, but the detailed information of the image is not properly preserved. Based on this algorithm, in view of the incompleteness of the existing fuzzy clustering algorithm [38-43], this paper further improves the existing clustering algorithm to enhance the anti-noise robustness of the original algorithm and segmentation accuracy. This paper introduces an iterative self-learning method to calculate the different 
weights of pixel information and neighborhood information, and solves the problem that the FLICMLNLI algorithm neglects to assign the same weight to the pixel information and the neighborhood information. The three innovations of the proposed robust self-learning weighted fuzzy clustering algorithm are as follows:

(1) The improved algorithm combines the local and non-local information of pixels and adopts a more reasonable correlation calculation model to achieve a better image segmentation effect.

(2) The improved algorithm can calculate the distance from the pixel to the cluster center more reasonably, because the original information of the pixel itself and the local information of the pixel must not be equally important. Through this improvement, the algorithm can eliminate most of the noise and retain a considerable degree of detailed information. It has better performance than FLICMLNLI algorithm.

(3) The improved algorithm solves the problem that the existing robust fuzzy clustering with spatial information constraints cannot automatically select the weighted factor. The algorithm solves this problem by obtaining weights through continuous iterative selflearning methods in the process of clustering.

The structure of this article is described as follows: The second section introduces the FCM algorithm and other classic algorithms; the third section analyzes the FLICMLNLI algorithm in detail; the fourth section presents a self-learning weighted distance metric, embedding improved distance measure into the objective function and gives the corresponding derivation and proof of the convergence process; the fifth part compares the segmentation results of the algorithm in this paper with the classic algorithm and the latest algorithm, and displays the comparison result graph and performance indicators; the final section gives the conclusion of this article.

\section{Image fuzzy clustering algorithm}

\subsection{FCM}

FCM algorithm is a fuzzy clustering algorithm based on objective function, which is mainly used for data clustering analysis. The theory is mature and widely used. It is an excellent clustering algorithm [44].

Suppose $X=\left\{x_{i}, i=1,2, \cdots\right\}$ represents a gray image to be segmented. Where $x_{i}$ is the intensity of the ith pixel, $i$ is the pixel index, $n$ is the total number of pixels.
The widely used optimization model of classic FCM can be expressed as follows:

$\min _{m}(U, V)=\sum_{i=1}^{n} \sum_{j=1}^{c} u_{i j}^{m} d^{2}\left(x_{i}, v_{j}\right)$

The constraints are: $0 \leq u_{i j} \leq 1, \quad \sum_{j=1}^{c} u_{i j}=1,0<$ $\sum_{i=1}^{n} u_{i j}<n . \quad 1 \leq i \leq n, 1 \leq j \leq c$.

Where $n, c, x_{i}$ and $v_{j}$ are the number of samples, the number of clusters, the ith sample in data set $X$ and the clustering center of the $j t h$ cluster respectively. $U=\left(u_{i j}\right)_{n \times c}$ is a fuzzy partition matrix, $u_{i j}$ denotes the membership of sample $x_{i}$ belonging to the $j$ th cluster. $d^{2}\left(x_{i}, v_{j}\right)=\left\|x_{i}-v_{j}\right\|^{2}$ represents the squared Euclidean distance between the sample $x_{i}$ and the clustering center $v_{j}$. $m \in[1,+\infty)$ is a fuzzy factor with the typical value of $1.5,2.0$ and 2.5, generally taking $m=2$. Using the Lagrange multiplier method to solve the optimization model (1), the membership degree $u_{i j}$ and clustering center $v_{j}$ can be obtained as follows:

$u_{i j}=1 / \sum_{k=1}^{c}\left(\frac{d^{2}\left(x_{i}, v_{j}\right)}{d^{2}\left(x_{i}, v_{k}\right)}\right)^{1 /(m-1)}$ and $\quad v_{j}=\sum_{i=1}^{n} u_{i j}^{m} x_{i} / \sum_{i=1}^{n} u_{i j}^{m}$

The algorithm is simple in principle and fast in operation, but it is sensitive to noise and outliers. Therefore, since its proposal, many researchers have devoted themselves to improving the algorithm. In this paper, the definitions of mathematical symbols $n, c, v, u, d, m$ and $x$ are consistent with this section.

\subsection{BCFCM}

FCM does not consider the spatial information of pixels but treats all pixels as isolated pixels. Generally, an image contains rich spatial information, such as local neighborhood means, median information and non-local spatial information. Therefore, it is of great significance to use spatial information to guide pixel clustering for image segmentation. In view of this, many scholars combine spatial neighborhood information with FCM and have obtained a series of corresponding improved algorithms.

Ahmed et al. [45] by introducing local spatial constraints in FCM, the BCFCM algorithm is proposed, which can obtain satisfactory results and reduce the influence of noise 
on the segmentation results. The optimization model of $\mathrm{BCFCM}$ is expressed as follows:

$$
\min J_{m}(U, V)=\sum_{i=1}^{n} \sum_{j=1}^{c} u_{i j}^{m} d_{i j}^{2}+\frac{\alpha}{N_{R}} \sum_{i=1}^{n} \sum_{j=1}^{c} u_{i j}^{m} \sum_{r \in N_{i}} d_{r j}^{2}
$$

Where $d_{i j}^{2}=\left\|x_{i}-v_{j}\right\|^{2}, N_{i}$ is the collection of adjacent pixels surrounding the ith pixel. $N_{R}$ is the number of neighboring pixels. The neighbors effect term is controlled by the parameter $\alpha$. Controlling the influence of neighboring pixels on clustering needs to be verified by experiment and error. The constraints are the same as (1). Solve (3) with Lagrange multiplier method, the membership degree $u_{i j}$ and clustering center $v_{j}$ can be obtained as follows:

$$
u_{i j}=\frac{\left(d_{i j}^{2}+\frac{\alpha}{N_{R}} \sum_{r \in N_{i}} d_{r j}^{2}\right)^{-1 /(m-1)}}{\sum_{k=1}^{c}\left(d_{i k}^{2}+\frac{\alpha}{N_{R}} \sum_{r \in N_{i}} d_{r k}^{2}\right)^{-1 /(m-1)}} \quad \text { and } \quad v_{j}=\frac{\sum_{i=1}^{n} u_{i j}^{m}\left(x_{i}+\frac{\alpha}{N_{R}} \sum_{r \in N_{i}} x_{r}\right)}{(1+\alpha) \sum_{i=1}^{n} u_{i j}^{m}}
$$

\subsection{EnFCM}

In order to improve the computational efficiency of the FCM algorithm, Szilagyi et al. [31] proposed an enhanced FCM, and its optimization model is expressed as follows:

$\min J_{m}(U, V)=\sum_{i=1}^{n} \sum_{j=1}^{c} \theta_{i} u_{i j}^{m}\left(\xi_{i}-v_{j}\right)^{2}$

Where $\xi_{i}=\frac{1}{1+\alpha}\left(x_{i}+\frac{\alpha}{N_{R}} \sum_{r \in N_{i}} x_{r}\right)$, The parameter $\alpha$ controls the intensity of the neighboring effect. $\theta_{i}$ represents the number of voxels from the whole stack of slices. The membership degree $u_{i j}$ and clustering center $v_{j}$ can be obtained as follows:

$$
u_{i j}=1 / \sum_{k=1}^{c}\left(\frac{\left(\xi_{i}-v_{j}\right)^{2}}{\left(\xi_{i}-v_{k}\right)^{2}}\right)^{1 /(m-1)} \text { and } v_{j}=\sum_{i=1}^{n} \theta_{i} u_{i j}^{m} \xi_{i} / \sum_{i=1}^{n} \theta_{i} u_{i j}^{m}
$$

\subsection{FLICM}

Krinidi et al. [33] proposed the FLICM algorithm by defining the restrictive relationship between the membership of the center pixel and the neighboring pixels, which effectively solved the problem that traditional fuzzy clustering algorithms are sensitive to noise and outliers. The optimization model of the FLICM algorithm is defined as:

$$
\min J_{m}(U, V)=\sum_{i=1}^{n} \sum_{j=1}^{c}\left(u_{i j}^{m}\left\|x_{i}-v_{j}\right\|^{2}+G_{i j}\right)
$$

Where $j$ is the category index. $c$ is the current number of clusters. $u_{i j}$ is the fuzzy membership function, which represents the degree to which the ith pixel belongs to the $j$ th category. $m$ is the fuzzy degree of the algorithm. $v_{j}$ is the cluster center of the $k t h$ cluster. $\left\|x_{i}-v_{j}\right\|^{2}$ is the Euclidean distance between the $i t h$ pixel and the $j t h$ cluster center. $G_{i j}$ is the blur factor can be defined as:

$$
G_{i j}=\sum_{\substack{i^{\prime} \in N_{i} \\ i^{\prime} \neq i}} \frac{1}{d_{i i^{\prime}}+1}\left(1-u_{i^{\prime} j}\right)^{m}\left\|x_{i^{\prime}}-v_{j}\right\|^{2}
$$

Where $N_{i}$ is the collection of adjacent pixels surrounding the $i t h$ pixel, $i^{\prime}$ is the index for neighborhood pixels, $d_{i i^{\prime}}$ is the spatial distance between the $i t h$ pixel and the $i^{\prime} t h$ cluster center. $G$ in this paper is consistent with this section. By minimizing the objective function, its membership degree $u_{i j}$ and clustering center $v_{j}$ can be obtained as follows:

$$
u_{i j}=1 / \sum_{k=1}^{c}\left(\frac{\left\|x_{i}-v_{j}\right\|^{2}+G_{i j}}{\left\|x_{i}-v_{k}\right\|^{2}+G_{i k}}\right)^{1 /(m-1)} \text { and } v_{j}=\frac{\sum_{i=1}^{n} u_{i j}^{m} x_{i}}{\sum_{i=1}^{n} u_{i j}^{m}}(9)
$$

In the objective function of the FLICM algorithm, the fuzzy factor calculation method shown in (8) ensures that the membership degrees of the pixels in the local window are similar. This method can reduce the influence of noise on the image. However, it is unreasonable to treat adjacent pixels as one category in general. When the noise level is high, the segmentation effect will be worse. The way to improve this problem is to ensure that similar pixels belong to the same cluster and not limited to adjacent pixels based on the membership of similar pixels. In addition, applying Euclidean distance in (8) will exclude pixels farther from the center pixel, which will further reduce performance.

\section{New image fuzzy clustering and its robust model}

The closer the two pixels are, it does not mean that the correlation between the two pixels is high. Therefore, the smaller the distance value, the greater the correlation calculation method is wrong. This paper adopts a more reasonable pixel correlation calculation model, which measures the correlation in the form of considering adjacent image blocks with different weights around the pixels. The specific calculation process of the correlation model is shown in Algorithm 1. 
Algorithm 1 Correlation model calculation process.

Input: The image $I$, and two parameters $\alpha, \gamma$

Output: Relevance between the central pixel $p$ and the pixels in the searching window.

Step 1: For any pixel $p$ in the image, construct image patches $X_{p}$.

Step 2: Retrieve the difference between corresponding patches in different directions:

$d_{p}(q)=\left(1 /\left|N_{p}\right|\right) \cdot \sum\left|X_{p}-X_{q}\right|$, in which $N_{p}$ is the set of neighboring pixels with

cardinality of $\left|N_{p}\right|$.

Step 3: Retrieve the weights in different directions:

$w_{p}(q)=\exp \left(-\alpha d_{p}(q)\right) / \sum_{q \in N_{p}} \exp \left(-\alpha d_{p}(q)\right)$

Step 4: Retrieve the weighted distance in different directions:

$d_{p}^{w}(q)=\left(1 /\left|N_{p}\right|\right) \cdot \sum_{q}\left(\omega_{p} \otimes\left|X_{p}-X_{q}\right|\right)$, where $\otimes$ is the dot product for two vectors.

Step 5: Retrieve the relevance between corresponding pixels:

$s(p, q)=\exp \left(-\gamma d_{p}^{\omega}(q)\right)$

In FLICMLNLI algorithm, the cluster center $v_{j}$ becomes a vector $\left(v_{j}, v_{j}^{N}\right)$. The blur factor is modified to:

$G_{i j}^{\prime}=\sum_{k \in W_{i}^{r}} s(i, k)\left(1-u_{j k}\right)^{m} d_{k j}^{2}$

Where $d_{k j}=\sqrt{\left(x_{k}-v_{j}\right)^{2}+\left\|x_{k}^{N}-v_{j}^{N}\right\|^{2}}, W_{i}^{r}$ represents the non-local information of the ith pixel. The corrected blur factor $G_{i j}^{\prime}$ can classify the center pixel and similar pixels (not all pixels) in the search window into the same category. The objective function of FLICMLNLI is as follows:

$J_{m}(U, V)=\sum_{i=1}^{n} \sum_{j=1}^{c}\left[u_{i j}^{m}\left(x_{i}-v_{j}\right)^{2}+\left\|x_{i}^{N}-v_{j}^{N}\right\|^{2}+G_{i j}^{\prime}\right]$

FLICMLNLI algorithm improves the correlation calculation and fuzzy factor calculation on the basis of FLICM algorithm, which improves the robustness of the algorithm. In addition, according to $\sum_{\mathrm{j}=1}^{c} u_{i j}=1$. Lagrange multiplier method (LMM) is used to minimize (11). By updating the clustering vector $\left(v_{j}, v_{j}^{N}\right)$ and the degree of membership $u_{i j}$, the objective function convergence and image segmentation are finally achieved. Algorithm 2 gives the detailed information of FLICMLNLI.
Algorithm 2 FLICMLNLI workflow.

Input: The image $I$, and three parameters: $c$, MaxIter Num and $\varepsilon$.

Output: Segmented image

1.Compute the neighboring information of pixels in the given image, denoted as $I^{N}$;

2. Initialized the partition matrix $U(0)$ and cluster center $V(0)$

by $\mathrm{FCM}$ algorithm, subject to $u_{i j}(0) \in[0,1]$ and $\sum_{j=1}^{c} u_{i j}(0)=1$;

$3 . t=0$;

4.for $t \leq$ maxIterNum do

5. $\quad$ Replace $v_{j}(t+1) \quad$ with $v_{j}(t+1)=\sum_{i=1}^{n} u_{i j}^{m}(t) x_{i} / \sum_{i=1}^{n} u_{i j}^{m}(t)$;

6. Replace $v_{j}^{N}(t+1) \quad$ with $v_{j}^{N}(t+1)=\sum_{i=1}^{n} u_{i j}^{m}(t) x_{i}^{N} / \sum_{i=1}^{n} u_{i j}^{m}(t)$;

7. Compute the distance between pixels and clusters according to

$d_{i j}(t+1)=\sqrt{\left(x_{i}-v_{j}(t+1)\right)^{2}+\left\|x_{i}^{N}-v_{j}^{N}(t+1)\right\|^{2}}$

8. Calculate the corrected blur factor $G_{i j}^{\prime}(t+1)$ with Eq.(10);

replace the fuzzy matrix $U(t+1)$ with

$u_{i j}(t+1)=1 / \sum_{k=1}^{c}\left(\frac{\left(x_{i}-v_{j}(t+1)\right)^{2}+\left\|x_{i}^{N}-v_{j}^{N}(t+1)\right\|^{2}+G_{i j}^{\prime}(t+1)}{\left(x_{i}-v_{k}(t+1)\right)^{2}+\left\|x_{i}^{N}-v_{k}^{N}(t+1)\right\|^{2}+G_{i k}^{\prime}(t+1)}\right)^{1 /(m-1)}$

10. If $\max \{\|U(t+1)-U(t)\|\}<\varepsilon$ then exit for;

11. $t=t+1$

12.The $j t h$ pixel is classified into the $k t h$ category, where $k=\arg \max \left\{u_{i j}\right\}$

FLICMLNLI algorithm is a relatively advanced fuzzy clustering algorithm at present. The correlation model proposed by this algorithm makes full use of the non-local information of pixels and introduces the local information when calculating the distance between pixels and the clustering center.

\section{Proposed algorithm}

FLICMLNLI algorithm introduces local information and non-local information, which improves the robustness of the algorithm to some extent, but there are also some problems. It is obviously unreasonable to assume that the original pixel information has the same importance as the neighborhood information when calculating the distance. Based on FLICMLNLI algorithm, the proposed algorithm retains the correlation model, introduces selflearning method, calculate the different weights of the 
original information and the neighborhood information when calculating the distance, and improves the algorithm to further improve the robustness of the algorithm.

As shown in Fig. 1, the algorithm proposed in this paper combines the current pixel information and its neighborhood information to obtain the latest weighted Euclidean distance, and introduces the current pixel information and non-local information at the same time. The latest local factors are proposed after fusion. The improved objective function is obtained by combining the traditional algorithm with the innovative local factor, the new cluster centers and membership degree matrix is obtained, through self-learning method constantly iterative calculation, each iteration to get the weight corresponding to the current pixel and its neighborhood information then get the distance measure, to achieve the optimal segmentation result.

The improved algorithm has two advantages. On one hand, it has obvious combinational novelty. In this paper, several concepts, methods, techniques and components, such as image segmentation, fuzzy clustering, distance measurement, self-learning, non-local information and noisy image are skillfully combined, which is a breakthrough. On the other hand, it breaks the inherent calculation mode of distance measurement in the past, gives different weights to the two which have different contributions to the calculation distance, and adopts the selflearning calculation method, which provides a brand new idea for the future research work.

\subsection{Preliminary knowledge}

In the article by Xinmin Tao [46], in order to express fuzzy clustering based on maximum entropy, the entropy criterion is first introduced. Let $X$ be a random variable with probability mass function $P(x)=P(X=x)$, and set of possible values $\left\{x_{i}, i=1, \ldots, n\right\}$. The entropy of random variable $X$ is denoted by $H(X)$ and is defined by $H(X)=-\sum P(x) \log P(x)$. Therefore, the structure of maximum entropy inference based on grades of membership $\left\{u_{i j}\right\}$ maximizes $\left\{-\sum_{i=1}^{n} \sum_{j=1}^{c} u_{i j} \log u_{i j}\right\}$. Applied to the FCM algorithm, the FCM algorithm requires finding a set of $\left\{u_{i k}\right\}$ to minimize the loss function of the normalization constraint. The maximum fuzzy clustering based on entropy is realized by using $L_{1}$-norm space and its goal is to maximize $\left\{-\sum_{i=1}^{n} \sum_{j=1}^{c} u_{i j} \log u_{i j}\right\}$ with respect to $L=\sum_{i=1}^{n} \sum_{j=1}^{c} \sum_{x=1}^{z} u_{i j}\left\|x_{i x}-v_{j x}\right\|$ and the normalization constraint. Here, $L$ is a loss function. To maximize $\left\{-\sum_{i=1}^{n} \sum_{j=1}^{c} u_{i j} \log u_{i j}\right\}$ subject to the above conditions, we use the Lagrange multiplier rule. The Lagrange function is:

$$
\begin{aligned}
L\left(u_{i j}, v_{j x}, \sigma, \lambda\right)= & -\sum_{i=1}^{n} \sum_{j=1}^{c} u_{i j} \log u_{i j}+\sigma\left(\sum_{i=1}^{n} \sum_{j=1}^{c} u_{i j} d_{i j}-L\right) \\
& +\lambda\left(\sum_{j=1}^{c} u_{i j}-1\right)
\end{aligned}
$$

Where $d_{i j}=\sum_{x=1}^{z}\left\|x_{i x}-v_{j x}\right\|$. Thus we see that in the maximum entropy-based reasoning, fuzzy clustering problem into a set of prototype, so the loss function and membership distribution that satisfy the normalization constraint and the maximum Lagrangian function are minimized.

In the article by Vikas Singh [47], by considering the different contribution of each feature in each cluster, a new entropy-based variable feature weighted fuzzy mean clustering algorithm was proposed. In this method, the weighted fuzzy mean algorithm is combined with membership entropy and feature weighted entropy at the same time. As defined in (18), the first item measures the difference between samples within a class, the second item measures the membership entropy between samples and classes in the clustering process, and the third item, the
Fig. 1 The framework of the proposed algorithm

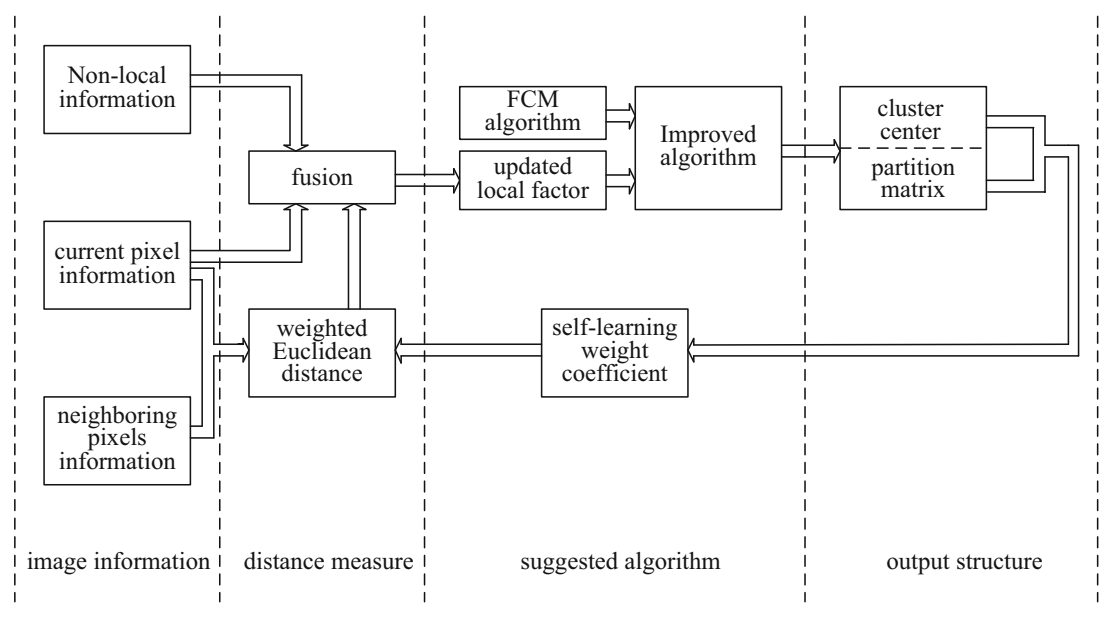


entropy of feature weights, represents clustering. The degree of certainty of features in class recognition.

Consider a data matrix $X=\left[X_{1}, X_{2}, \ldots X_{n}\right] \in R^{q \times n}, q$ and $n$ are the number of features and number of samples respectively. Here, $X_{i}=\left[x_{i 1}, x_{i 2}, \ldots, x_{i q}\right]^{\prime} \in R^{q}$ is the $i t h$ sample in the data matrix. To group the data matrix $X$ into $c$ number of clusters, the following objective function can be minimized:

$$
\begin{aligned}
J(U, V, W)= & \sum_{i=1}^{n} \sum_{j=1}^{c} \sum_{e=1}^{q} u_{i j} w_{j e} D_{i j}^{e}+\sum_{i=1}^{n} \mu_{i} \sum_{j=1}^{c} u_{i j} \log u_{i j} \\
& +\sum_{j=1}^{c} \eta_{j} \sum_{e=1}^{q} w_{j e} \log w_{j e}
\end{aligned}
$$

The constraints are: $\sum_{j=1}^{c} u_{i j}=1, \quad \sum_{e=1}^{q} w_{j e}=1 ; \quad 0 \leq$ $u_{i j}, w_{j e} \leq 1,1 \leq i \leq n, 1 \leq j \leq k$.

Where $D_{i j}^{e}=\left(x_{i e}-v_{j e}\right)^{2}$, is the Euclidean distance metric between $i t h$ sample and $j$ th cluster, $x_{i e}$ is the value of $l t h$ feature of $i t h$ sample, $v_{j e}$ is the value of $l t h$ feature of $j t h$ cluster, $U=\left[u_{i j}\right]$ is the $n \times c$ fuzzy partition matrix, $u_{i j}$ is the membership degree value of $j t h$ cluster of $i t h$ sample, $V=\left[v_{j e}\right]$ is the $c \times p$ matrix having the cluster centers, $W=\left[w_{j e}\right]$ is an $c \times p$ weight matrix, $w_{j e}$ is the weight value of $l t h$ feature to $j t h$ cluster, $\eta_{i}$ and $\mu_{j}$ are the input parameters used to control the fuzzy partition and feature weight respectively.

The objective function as shown in (12) is a constrained nonlinear optimization problem, the solution of which is unknown. The main aim is to minimize $J$ with respect to $U$, $V$ and $W$ using alternating optimization method.

\subsection{Optimization model and solution}

Combining the maximum entropy fuzzy clustering and sample attribute weighting in Xinmin Tao and Vikas Singh's article to construct the optimization model of the algorithm proposed in this paper. In the new algorithm proposed in this paper, instead of maximizing the maximum entropy reasoning structure based on the degree of membership, the maximum entropy reasoning structure based on the weight is used to maximize the maximum entropy reasoning structure, so $\left\{-\sum_{i=1}^{n} \sum_{j=1}^{c} u_{i j} \log u_{i j}\right\}$ is rewritten as $\left\{\sum_{w=1}^{2} \omega_{w} \ln \omega_{w}\right\}$. The optimization model of the proposed algorithm is expressed as follows:

$\min J_{m}(U, V)=\sum_{i=1}^{n} \sum_{j=1}^{c} u_{i j}^{m}\left(d_{i j}^{2}+\tilde{G}_{i j}\right)+\rho \sum_{w=1}^{2} \omega_{w} \ln \omega_{w}$

In (14), the first term measures the difference between samples within the same class, and the entropy of the second term feature weight indicates the degree of certainty of features in cluster recognition.

The constraints are: $\sum_{j=1}^{c} u_{i j}=1,0 \leq u_{i j} \leq 1,1 \leq i \leq$ $n$ and $\sum_{w=1}^{2} \omega_{w}=1,0 \leq \omega_{w} \leq 1$.

Where $d_{i j}=\sqrt{\omega_{1}\left(x_{i}-v_{j}\right)^{2}+\omega_{2}\left\|x_{i}^{N}-v_{j}^{N}\right\|^{2}}$, The improved distance measurement formula contains the original pixel information and the local pixel information and their weights are different, which can better distinguish noise and image details. The improved blur factor is as follows:

$$
\tilde{G}_{i j}=\sum_{k \in N_{i}} s u \cdot d_{k j}^{2}
$$

Where $s u=s(k, i)\left(1-u_{k j}\right)^{m}$, the followings are the same. In (15), non-local information of pixels is used when calculating pixel correlation. The same applies to the Lagrange multiplier rule, the Lagrange function is:

$$
\begin{aligned}
L= & \sum_{i=1}^{n} \sum_{j=1}^{c} u_{i j}^{m}\left(d_{i j}^{2}+\tilde{G}_{i j}\right)+\rho \sum_{w=1}^{2} \omega_{w} \ln \omega_{w} \\
& +\sum_{i=1}^{n} \lambda_{i}\left(1-\sum_{j=1}^{c} u_{i j}\right)+w\left(1-\omega_{1}-\omega_{2}\right)
\end{aligned}
$$

Also use alternate optimization method to minimize.

First, use weights to minimize the process as follows:

$$
\begin{aligned}
\frac{\partial L}{\partial \omega_{1}}= & \sum_{i=1}^{n} \sum_{j=1}^{c}\left[u_{i j}^{m}\left(\left(x_{i}-v_{j}\right)^{2}+\sum_{k \in N_{i}} s u \cdot\left(x_{k}-v_{j}\right)^{2}\right)\right] \\
& +\rho\left(1+\ln \omega_{1}\right)-w=0
\end{aligned}
$$

$$
\begin{aligned}
\frac{\partial L}{\partial \omega_{2}}= & \sum_{i=1}^{n} \sum_{j=1}^{c}\left[u_{i j}^{m}\left(\left\|x_{i}^{N}-v_{j}^{N}\right\|^{2}+\sum_{k \in N_{i}} s u \cdot\left\|x_{k}^{N}-v_{j}^{N}\right\|^{2}\right)\right] \\
& +\rho\left(1+\ln \omega_{2}\right)-w=0
\end{aligned}
$$

Then get:

$\omega_{1}=\exp \left(\frac{\sum_{i=1}^{n} \sum_{j=1}^{c}\left[u_{i j}^{m}\left(\left(x_{i}-v_{j}\right)^{2}+\sum_{k \in N_{i}} s u \cdot\left(x_{k}-v_{j}\right)^{2}\right)\right]+\rho}{-\rho}\right)$

$\omega_{2}=\exp \left(\frac{\sum_{i=1}^{n} \sum_{j=1}^{c}\left[u_{i j}^{m}\left(\left\|x_{i}^{N}-v_{j}^{N}\right\|^{2}+\sum_{k \in N_{i}} s u \cdot\left\|x_{k}^{N}-v_{j}^{N}\right\|^{2}\right)\right]+\rho}{-\rho}\right)(20)$

Because of $\omega_{1}+\omega_{2}=1$, The final weights are $\omega_{1}^{\prime}=$ $\omega_{1} /\left(\omega_{1}+\omega_{2}\right)$ and $\omega_{2}^{\prime}=\omega_{2} /\left(\omega_{1}+\omega_{2}\right)$.

Second, the process of minimizing $L$ with $V$ is as follows: 


$$
\begin{gathered}
\frac{\partial L}{\partial v_{j}} \quad=\quad \sum_{i=1}^{n} u_{i j}^{m}\left[-2 \omega_{1}\left(x_{i}-v_{j}\right)-\right. \\
\left.2 \omega_{1} \sum_{k \in N_{i}} s u \cdot\left(x_{k}-v_{j}\right)\right]=0 \\
\Rightarrow v_{j}\left[\sum_{i=1}^{n} u_{i j}^{m}\left(1+\sum_{k \in N_{i}} s u\right)\right]=\sum_{i=1}^{n} u_{i j}^{m}\left(x_{i}+\sum_{k \in N_{i}} s u \cdot x_{k}\right)
\end{gathered}
$$

The calculation formula for obtaining cluster centers is as follows:

$$
v_{j}=\sum_{i=1}^{n} u_{i j}^{m}\left(x_{i}+\sum_{k \in N_{i}} s u \cdot x_{k}\right) / \sum_{i=1}^{n} u_{i j}^{m}\left(1+\sum_{k \in N_{i}} s u\right)
$$

Third, because of $\left\|x_{i}^{N}-v_{j}^{N}\right\|^{2}=\sum_{l=1}^{p}\left(x_{i l}^{N}-v_{j l}^{N}\right)^{2}$, then $\frac{\partial L}{\partial v_{j}^{N}}=\sum_{i=1}^{n} u_{i j}^{m}\left[-2 \omega_{2}\left(\sum_{l=1}^{p} x_{i l}^{N}-v_{j l}^{N}\right)-2 \omega_{2} \sum_{k \in N_{i}} s u \cdot\left(\sum_{l=1}^{p} x_{k l}^{N}-v_{j l}^{N}\right)\right]=0$ $\Rightarrow v_{j}^{N}\left[\sum_{i=1}^{n} u_{i j}^{m}\left(1+\sum_{k \in N_{i}} s u\right)\right]=\sum_{i=1}^{n} u_{i j}^{m}\left(x_{i}^{N}+\sum_{k \in N_{i}} s u \cdot x_{k}^{N}\right)$

$$
v_{j}^{N}=\sum_{i=1}^{n} u_{i j}^{m}\left(x_{i}^{N}+\sum_{k \in N_{i}} s u \cdot x_{k}^{N}\right) / \sum_{i=1}^{n} u_{i j}^{m}\left(1+\sum_{k \in N_{i}} s u\right)(22)
$$

Last, the process of minimizing $L$ with $U$ is as follows: $\frac{\partial L}{\partial u_{i j}}=m u_{i j}^{m-1}\left(d_{i j}^{2}+\tilde{G}_{i j}\right)-\lambda_{i}=0 \Rightarrow$ $u_{i j}=\left(\frac{\lambda_{i}}{m\left(d_{i j}^{2}+\tilde{G}_{i j}\right)}\right)^{\frac{1}{m-1}} \Rightarrow 1=\lambda_{i}^{\frac{1}{m-1}}\left(m \sum_{r=1}^{c}\left(d_{i j}^{2}+\tilde{G}_{i r}\right)\right)^{-\frac{1}{m-1}}$ $\Rightarrow \lambda_{i}^{\frac{1}{m-1}}=\left(m \sum_{r=1}^{c}\left(d_{i j}^{2}+\tilde{G}_{i r}\right)\right)^{\frac{1}{m-1}}$

The calculation formula for the membership degree is as follows:

$$
u_{i j}=1 / \sum_{k=1}^{c}\left(\frac{\left(d_{i j}^{2}+\tilde{G}_{i j}\right)}{\left(d_{i k}^{2}+\tilde{G}_{i k}\right)}\right)^{1 /(m-1)}
$$

The improved algorithm flow is shown in Algorithm 3.

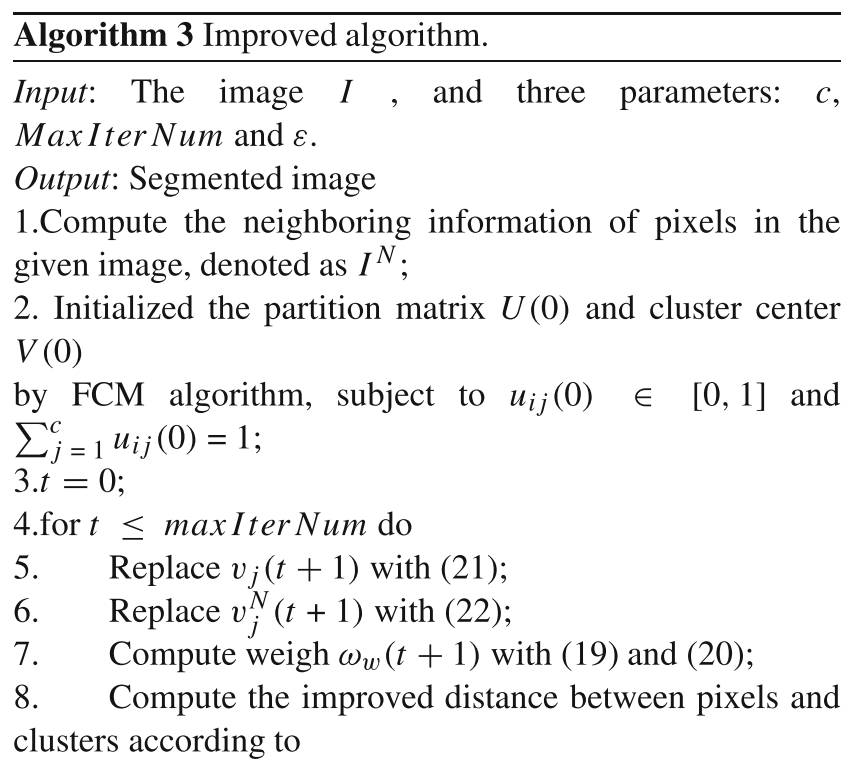
$d_{i j}(t+1)=\sqrt{\omega_{1}(t+1)\left(x_{i}-v_{j}(t+1)\right)^{2}+\omega_{2}(t+1)\left\|x_{i}^{N}-v_{j}^{N}(t+1)\right\|^{2}}$

9. Calculate the corrected blur $\tilde{G}_{i j}(t+1)$ factorwith (22);

10. Replace the fuzzy matrix $U(t+1)$ with (23);

11. If $\max \{\|U(t+1)-U(t)\|\}<\varepsilon$ then exit for;

12. $t=t+1$

13.The $j$ th pixel is classified into the $k t h$ category, where $k=\arg \max \left\{u_{i j}\right\}$

The improved algorithm uses the non-local information of pixels when calculating the correlation between pixels, and the original information and local information of pixels are used when calculating the distance between pixels and the cluster center, and the two play different roles in calculating the distance, so the weights are different. This improvement makes the proposed algorithm more reasonable and has better segmentation effect than FLICMLNLI algorithm.

\subsection{Proof of convergence}

\subsubsection{Convergence proof of $U$}

The objective function is expressed as follows:

$$
\begin{aligned}
& L(U)=\sum_{i=1}^{n} \sum_{j=1}^{c} u_{i j}^{m}\left(d_{i j}+\tilde{G}_{i j}\right)+\rho\left(\omega_{1} \ln \omega_{1}+\omega_{2} \ln \omega_{2}\right) \\
& +\sum_{i=1}^{n} \lambda_{i}\left(1-\sum_{j=1}^{c} u_{i j}\right)
\end{aligned}
$$


Where $\quad \tilde{G}_{i j}=\sum_{k \in N_{i}} s u \cdot d_{k j}^{2} \quad$ and $\quad d_{i j}=$ $\sqrt{\omega_{1}\left(x_{i}-v_{j}\right)^{2}+\omega_{2}\left\|x_{i}^{N}-v_{j}^{N}\right\|^{2}}, \omega_{1}$ and $\omega_{2}$ are constants. If $u^{*}$ is the minimum of $L(U)$, then $\frac{\partial L\left(u^{*}\right)}{\partial u_{i j}}=0$, that is $\frac{\partial L\left(u^{*}\right)}{\partial u_{i j}}=m u_{i j}^{m-1}\left(d_{i j}^{2}+\tilde{G}_{i j}\right)-\lambda_{i}=0$, the following formula can be obtained:

$u_{i j}^{*}=1 / \sum_{k=1}^{c}\left(\frac{\left(d_{i j}^{2}+\tilde{G}_{i j}\right)}{\left(d_{i k}^{2}+\tilde{G}_{i k}\right)}\right)^{1 /(m-1)}$

The calculation process of the Hessian matrix of $L(u)$ at $u=u^{*}$ is as follows:

$\frac{\partial}{\partial u_{a b}}\left(\frac{\partial L\left(u^{*}\right)}{\partial u_{i j}}\right)=\left\{\begin{array}{cccc}0, & a \neq i & \text { or } & b \neq j \\ m(m-1) u_{i j}^{m-2}\left(d_{i j}^{2}+\tilde{G}_{i j}\right) & a=i & \text { and } & b=j\end{array}\right.$

It can be found from (26) that the characteristic of the Hessian matrix of $L(u)$ at $u=u^{*}$ is that the diagonal elements of the matrix are greater than zero, and the nondiagonal elements are zero, which shows that the obtained Hessian matrix is a symmetric positive definite matrix, so $u^{*}$ is a local minimum of $L(U)$.

\subsubsection{Convergence proof of $V$}

The objective function is expressed as follows:

$$
\begin{aligned}
& L(V)=\sum_{i=1}^{n} \sum_{j=1}^{c} u_{i j}^{m}\left(d_{i j}+\tilde{G}_{i j}\right)+\rho\left(\omega_{1} \ln \omega_{1}+\omega_{2} \ln \omega_{2}\right) \\
& +\sum_{i=1}^{n} \lambda_{i}\left(1-\sum_{j=1}^{c} u_{i j}\right)
\end{aligned}
$$

Where $\quad \tilde{G}_{i j}=\sum_{k \in N_{i}} s u \cdot d_{k j}^{2}$ and $d_{i j}=$ $\sqrt{\omega_{1}\left(x_{i}-v_{j}\right)^{2}+\omega_{2}\left\|x_{i}^{N}-v_{j}^{N}\right\|^{2}}, \quad \omega_{1} \quad$ and $\quad \omega_{2}$ are constants. If $v^{*}$ is the minimum of $L(V)$, then $\frac{\partial L\left(v^{*}\right)}{\partial v_{j}}=0$, that is $\frac{\partial L\left(v^{*}\right)}{\partial v_{j}}=\sum_{i=1}^{n} u_{i j}^{m}\left[-2 \omega_{1}\left(x_{i}-v_{j}\right)-\right.$ $\left.2 \omega_{1} \sum_{k \in N_{i}} s u \cdot\left(x_{k}-v_{j}\right)\right]=0$, the following formula can be obtained:

$v_{j}^{*}=\sum_{i=1}^{n} u_{i j}^{m}\left(x_{i}+\sum_{k \in N_{i}} s u \cdot x_{k}\right) / \sum_{i=1}^{n} u_{i j}^{m}\left(1+\sum_{k \in N_{i}} s u\right)$

The calculation process of the Hessian matrix of $L(v)$ at $v=v^{*}$ is as follows:

$$
\frac{\partial}{\partial v_{k}}\left(\frac{\partial L\left(v^{*}\right)}{\partial v_{j}}\right)=\left\{\begin{array}{cl}
0, & k \neq j \\
\left.\sum_{i=1}^{n} u_{i j}^{m}\left[2 \omega_{1}+2 \omega_{1} \sum_{k \in N_{i}} s u\right)\right], & k=j
\end{array}\right.
$$

It can be found from (29) that the characteristic of the Hessian matrix of $L(v)$ at $v=v^{*}$ is that the diagonal elements of the matrix are greater than zero, and the nondiagonal elements are zero, which shows that the obtained Hessian matrix is a symmetric positive definite matrix, so $v^{*}$ is a local minimum of $L(V)$.

\subsubsection{Convergence proof of $V^{N}$}

The objective function is expressed as follows:

$$
\begin{aligned}
& L\left(V^{N}\right)=\sum_{i=1}^{n} \sum_{j=1}^{c} u_{i j}^{m}\left(d_{i j}+\tilde{G}_{i j}\right)+\rho\left(\omega_{1} \ln \omega_{1}+\omega_{2} \ln \omega_{2}\right) \\
& +\sum_{i=1}^{n} \lambda_{i}\left(1-\sum_{j=1}^{c} u_{i j}\right)
\end{aligned}
$$

Where $\tilde{G}_{i j}=\sum_{k \in N_{i}} s u \cdot d_{k j}^{2}$ and $d_{i j}=$ $\sqrt{\omega_{1}\left(x_{i}-v_{j}\right)^{2}+\omega_{2}\left\|x_{i}^{N}-v_{j}^{N}\right\|^{2}}, \omega_{1}$ and $\omega_{2}$ are constants. If $v_{j}^{N *}$ is the minimum of $L\left(V^{N}\right)$, then $\frac{\partial L\left(v^{N *}\right)}{\partial v_{j}^{N}}=0$ , that is $\frac{\partial L\left(v^{N *}\right)}{\partial v_{j}^{N}}=\sum_{i=1}^{n} u_{i j}^{m}\left[-2 \omega_{2}\left(\sum_{l=1}^{p} x_{i l}^{N}-v_{j l}^{N}\right)-\right.$ $\left.2 \omega_{2} \sum_{k \in N_{i}} s u \cdot\left(\sum_{l=1}^{p} x_{k l}^{N}-v_{j l}^{N}\right)\right]=0$, the following formula can be obtained:

$v_{j}^{N *}=\sum_{i=1}^{n} u_{i j}^{m}\left(x_{i}^{N}+\sum_{k \in N_{i}} s u \cdot x_{k}^{N}\right) / \sum_{i=1}^{n} u_{i j}^{m}\left(1+\sum_{k \in N_{i}} s u\right)$

The calculation process of the Hessian matrix of $L\left(v^{N}\right)$ at $v^{N}=v^{N *}$ is as follows:

$\frac{\partial}{\partial v_{k}^{N}}\left(\frac{\partial L\left(v^{N *}\right)}{\partial v_{j}^{N}}\right)=\left\{\begin{array}{cr}0, & k \neq j \\ \sum_{i=1}^{n} u_{i j}^{m}\left[2 p \omega_{2}+2 p \omega_{2} \sum_{k \in N_{i}} s u\right] & k=j\end{array}\right.$

It can be found from (32) that the characteristic of the Hessian matrix of $L(v)$ at $v=v^{*}$ is that the diagonal elements of the matrix are greater than zero, and the nondiagonal elements are zero, which shows that the obtained Hessian matrix is a symmetric positive definite matrix, so $v_{j}^{N *}$ is a local minimum of $L\left(V^{N}\right)$.

In conclusion, this section confirms the convergence of the algorithm proposed in this article from three aspects.

\section{Experiments and results}

\subsection{Evaluation index}

The performance of the comparison algorithm can be calculated by calculating the performance index. This section gives the calculation formula of each index. 
The calculation formula of partition coefficient $\left(V_{P C}\right)$ and partition entropy $\left(V_{P E}\right)$ are as follows:

$V_{P C}=\frac{1}{n} \sum_{i=1}^{n} \sum_{j=1}^{c} u_{i j}^{2}$

$V_{P E}=\frac{1}{n} \sum_{i=1}^{n} \sum_{j=1}^{c} u_{i j} \log \left(u_{i j}\right)$

The segmentation result with the smallest blur is the best, so the larger $V_{P C}$, the smaller $V_{P E}$ of the segmented image, the better the segmentation effect. In addition, there are four other indicators for comparing the segmented image and the ideal image of each algorithm. They are $\operatorname{accuracy}(A c c$.$) ,$ sensitivity (Sen.), specificity(Spe.), and precision(Pre.) [48].Their calculation formulas are as follows:

$$
\begin{aligned}
& \text { Acc. }=(T P+T N) /(T P+T N+F P+F N) \\
& \text { Sen. }=T P /(T P+F N) \\
& \text { Spe. }=T N /(T N+F P) \\
& \text { Pre. }=T P /(T P+F P)
\end{aligned}
$$

Among them, $P, N, T$ and $F$ represent positive, negative, true and false respectively. Accuracy is the ratio of pixels that correctly classify all pixels relative to all pixels. Sensitivity and specificity reveal the possibility of correctly classifying positive and negative pixels. Therefore, the values of these four measurement methods are between 0 and 1 . The greater the accuracy, sensitivity, specificity, and precision, the better the segmentation effect.

Misclassification error(ME) [49] as a quantitative evaluation index can objectively and quantitatively describe the effectiveness and robustness of the algorithm, which is:

$M E=\left(1-\sum_{i=1}^{c}\left|A_{i} \cap B_{i}\right| \cdot\left(\sum_{j=1}^{c}\left|B_{j}\right|\right)^{-1}\right) \times 100 \%$

Where $A_{i}$ represents the set of pixels belonging to the $i t h$ category in the ideal segmented image, $B_{j}$ represents the set of pixels belonging to the $j t h$ category in the real segmented image obtained by the algorithm. $c$ represents the number of classes. The smaller $M E$, the closer the segmentation result is to the ideal segmentation result, the better segmentation performance and vice versa.
Similarly, the peak signal-to-noise ratio(PSNR) [50], as a quantitative evaluation index of the robustness of the segmentation algorithm, is defined as:

$P S N R=10 \cdot \log 10\left(225^{2} / M S E\right)$

\subsection{Parameter selection}

In the experiment, it is very important to define the number of classes in advance, because different numbers of classes will present different details [51]. In our experiment, the number of categories in the composite image is known in advance. For other types of images, we determine the value of $c$ based on different segmentation tasks. The values of other parameters in the relevant algorithm are shown in Table 1. The parameters are obtained through many experiments. Each mathematical symbol in the Table 1 corresponds to the preceding sections.

\subsection{Experimental results and analysis}

In this section, different types of noise are used to destroy the original image in Fig. 2. Figure 2c-e are selected from BSDS500 gallery. Figure 2f-m are selected from MedPix database. The segmentation results of the improved algorithm and FCM, BCFCM, EnFCM, FLICM and FLICMLNLI are compared.

\subsubsection{Comparison of segmentation performance without noise}

As shown in Fig. 3a, three medical images were selected. Medical images often contain a lot of detailed information, and the detailed information of medical images needs to be preserved. The selected medical images were divided into three categories. Comparing Fig. 3c-h with b, it can be found that the algorithm proposed in this paper is closer to the ideal segmentation image and can better classify the details of the original image. On the contrary, the classification results of FLICMLNLI algorithm are relatively rough, ignoring many details and unable to recover the original image well. Furthermore, the evaluation indexes obtained by the

Table 1 Experimental parameters

\begin{tabular}{llllll}
\hline Algorithm & $m$ & $\varepsilon$ & $N_{R}$ & $\rho$ & $\alpha$ \\
\hline FCM & 2 & 0.01 & - & - & - \\
BCFCM & 2 & 0.01 & 9 & - & - \\
EnFCM & 2 & 0.01 & 9 & - & - \\
FLICM & 2 & 0.01 & 8 & - & - \\
FLICMLNLI & 2 & 0.01 & 81 & - & 0.05 \\
Improved & 2 & 0.01 & 81 & 0.499 \\
\end{tabular}


Fig. 2 Original image (a)-(b)

Synthetic image 1-2 (c)-(e)

Natural image 1-3 (f)-(m)

Medical image 1-8 (n) Remote sensing image

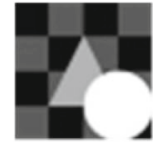

(a)

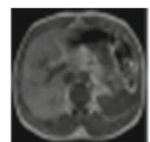

(h)

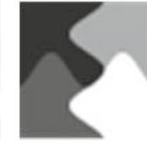

(b)

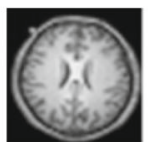

(i)

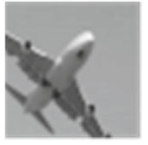

(c)

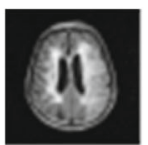

(j)

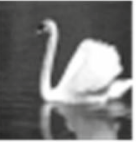

(d)

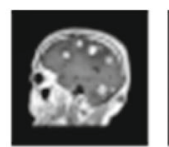

(k)

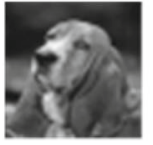

(e)

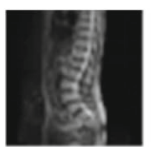

(1)

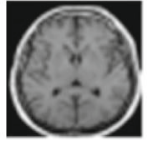

(f)

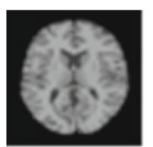

(m)

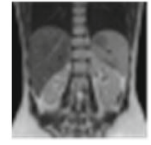

(g)

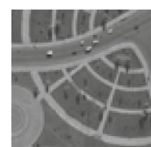

(n) segmentation of images by various algorithms are shown in Table 2. The comparison of all indexes also leads to the conclusion that the algorithm proposed in this paper is superior to other algorithms in the segmentation of original medical images.

\subsubsection{Comparison of segmentation performance with Gaussian noise}

Figure 4a contains three images, one natural image and two medical images. The first row of Fig. 4a is a swan image. Different algorithms are used to segment the swan image polluted by Gaussian noise with a normalized variance of 0.1 , and the segmentation result of the algorithm proposed in this paper is the closest to the ideal image. It is obvious that the algorithm proposed in this article has stronger anti-Gaussian noise resistance than other algorithms.

The second and third rows in Fig. 4a are two medical images with rich details, compare the segmentation results of various algorithms on medical images after they are contaminated by Gaussian noise. By comparing the result graph of each algorithm with the ideal image, it can be found that the algorithm proposed in this paper has the best restoration effect, which can not only remove most of the noise pollution, but also retain the details of the original image.

The performance indicators in Table 3 further confirm the statement that the algorithm performance in this paper is better. It shows that the algorithm in this paper achieves a better balance between noise removal and detail preservation. It has a strong ability to process medical images, which is more effective than other algorithm.

\subsubsection{Comparison of segmentation performance with Salt and Pepper noise}

Figure 5a shows three different types of original images, where the first row is a composite image, the second is

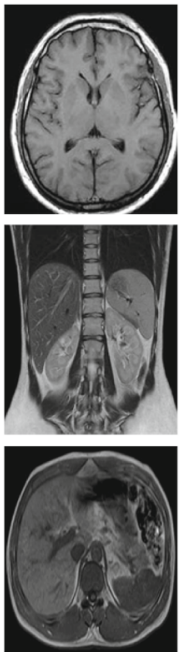

(a)
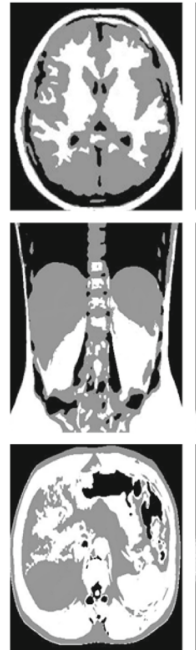

(b)
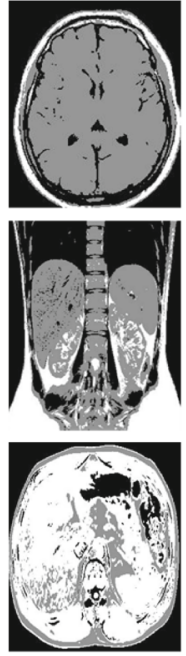

(c)
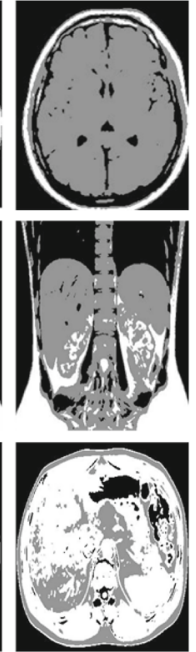

(d)
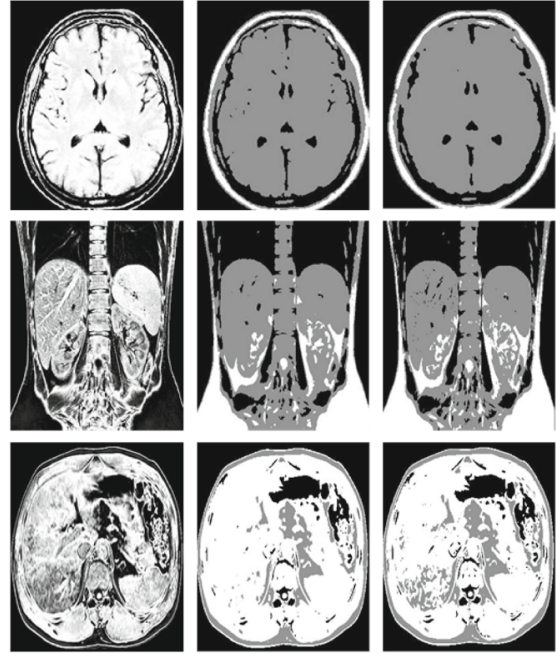

(e)

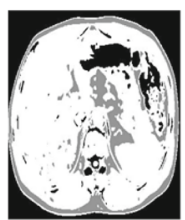

(f)
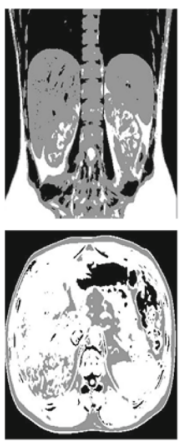

(g)
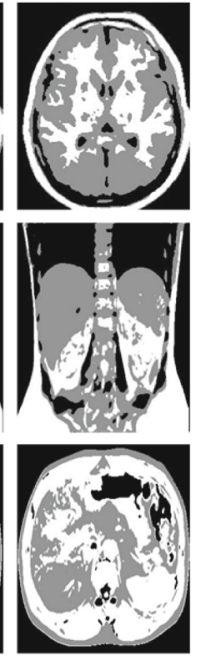

(h)

Fig. 3 The original image $(C=3)$ segmentation result map a Original image b Ideal image $\mathbf{c}$ FCM result $\mathbf{d}$ BCFCM result $\mathbf{e}$ EnFCM result $\mathbf{f}$ FLICM result $\mathbf{g}$ FLICMLNLI result $\mathbf{h}$ Improved result 
Table 2 The performance index of different algorithms to segment the original image

\begin{tabular}{|c|c|c|c|c|c|c|c|}
\hline Image & Index & FCM & BCFCM & EnFCM & FLICM & FLICMLNLI & Improved \\
\hline \multirow[t]{7}{*}{ Fig. 3 (row1) } & $V_{P C}$ & 0.9011 & 0.8297 & 0.9011 & 0.9151 & 0.8306 & 0.9667 \\
\hline & Acc. & 0.8294 & 0.9697 & 0.9537 & 0.9631 & 0.9467 & 0.9790 \\
\hline & Sen. & 0.7441 & 0.9717 & 0.9621 & 0.9591 & 0.9201 & 0.9785 \\
\hline & Spe. & 0.8720 & 0.9686 & 0.9533 & 0.9787 & 0.9600 & 0.9842 \\
\hline & Pre. & 0.7441 & 0.9394 & 0.9027 & 0.9443 & 0.9201 & 0.9685 \\
\hline & $P S N R$ & 11.6947 & 16.4088 & 15.4243 & 18.1091 & 17.0642 & 21.1398 \\
\hline & $M E$ & 0.2558 & 0.0443 & 0.0898 & 0.0693 & 0.0798 & 0.0314 \\
\hline \multirow[t]{7}{*}{ Fig. 3 (row2) } & $V_{P C}$ & 0.8396 & 0.7976 & 0.8395 & 0.9055 & 0.7872 & 0.9062 \\
\hline & Acc. & 0.8948 & 0.9680 & 0.9002 & 0.9627 & 0.9470 & 0.9715 \\
\hline & Sen. & 0.9021 & 0.9432 & 0.8526 & 0.9577 & 0.9230 & 0.9573 \\
\hline & Spe. & 0.9158 & 0.9704 & 0.9239 & 0.9321 & 0.9591 & 0.9786 \\
\hline & Pre. & 0.8509 & 0.9422 & 0.8486 & 0.8549 & 0.9186 & 0.9573 \\
\hline & $P S N R$ & 15.2521 & 17.5040 & 13.5027 & 15.5712 & 16.5664 & 19.7317 \\
\hline & $M E$ & 0.1098 & 0.0463 & 0.1496 & 0.0801 & 0.0793 & 0.0426 \\
\hline \multirow[t]{7}{*}{ Fig. 3 (row3) } & $V_{P C}$ & 0.8003 & 0.7469 & 0.8003 & 0.8823 & 0.8319 & 0.9684 \\
\hline & Acc. & 0.9101 & 0.9467 & 0.8581 & 0.9513 & 0.9491 & 0.9604 \\
\hline & Sen. & 0.9255 & 0.9321 & 0.7872 & 0.9622 & 0.9236 & 0.9606 \\
\hline & Spe. & 0.9545 & 0.9540 & 0.8936 & 0.9608 & 0.9618 & 0.9703 \\
\hline & Pre. & 0.8973 & 0.9103 & 0.7872 & 0.9311 & 0.9236 & 0.9406 \\
\hline & $P S N R$ & 12.0162 & 13.3631 & 10.1624 & 14.0121 & 13.4073 & 14.9600 \\
\hline & $M E$ & 0.0669 & 0.0797 & 0.2127 & 0.0677 & 0.0763 & 0.0593 \\
\hline
\end{tabular}

a natural image, and the third is a medical image. These three kinds of images are polluted by $20 \%$ Salt and Pepper noise, as shown in Fig. 5b. The segmentation results of different segmentation algorithms are shown in Fig. 5d-i. By comparing the segmentation results of different algorithms with the ideal image, it is found that the segmentation effect of the algorithm proposed in this paper is closer to the ideal effect. The results of calculating the corresponding evaluation indexes are shown in Table 4, and the algorithm in this paper has the best effect.

\subsubsection{Comparison of segmentation performance with Mixed noise}

Combine Fig. 6 and Table 5, improved algorithm also works best when processing images contaminated by Mixed noise.

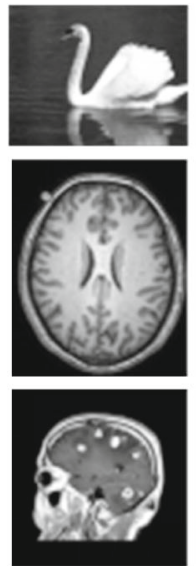

(a)
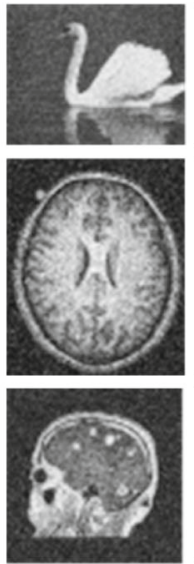

(b)
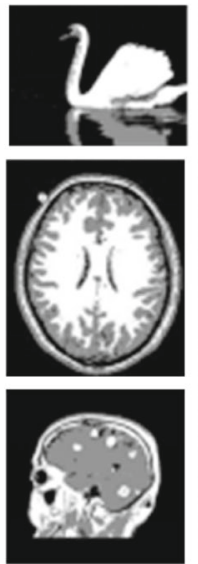

(c)

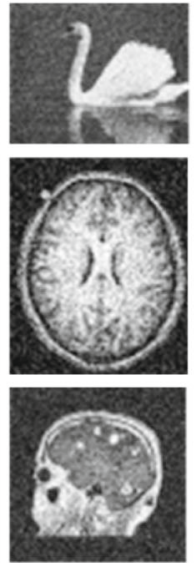

(d)

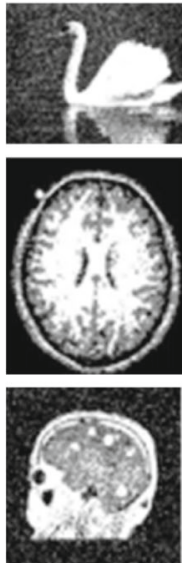

(e)
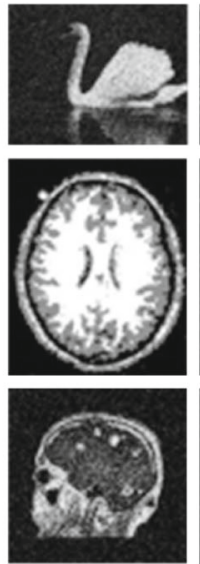

(f)

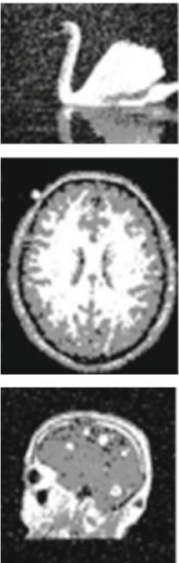

(g)
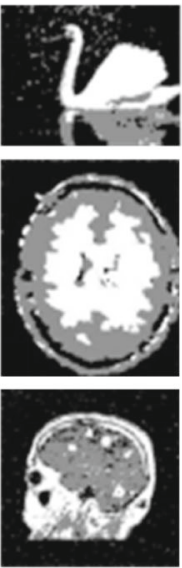

(h)
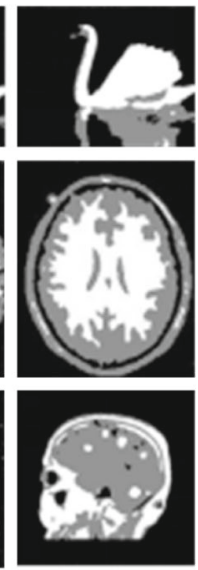

(i)

Fig. 4 The segmentation result of image polluted by Gaussian noise with a normalized variance of 0.1 a Original image b Noisy image $\mathbf{c}$ Ideal image d FCM result a BCFCM result f EnFCM result $\mathbf{g}$ FLICM result h FLICMLNLI result i Improved result 
Table 3 The performance index of different algorithms for segmenting original images polluted by Gaussian noise

\begin{tabular}{|c|c|c|c|c|c|c|c|}
\hline Image & Index & FCM & BCFCM & EnFCM & FLICM & FLICMLNLI & Improved \\
\hline \multirow[t]{7}{*}{ Fig. 4 (row1) } & $V_{P C}$ & 0.8288 & 0.8446 & 0.8025 & 0.6806 & 0.9518 & 0.9617 \\
\hline & $A c c$. & 0.7433 & 0.7928 & 0.8324 & 0.8686 & 0.9322 & 0.9480 \\
\hline & Sen. & 0.6149 & 0.6995 & 0.8123 & 0.8030 & 0.8983 & 0.9221 \\
\hline & Spe. & 0.8074 & 0.8394 & 0.8907 & 0.9015 & 0.9491 & 0.9610 \\
\hline & Pre. & 0.6149 & 0.6853 & 0.7145 & 0.8030 & 0.8983 & 0.9221 \\
\hline & $P S N R$ & 8.9927 & 10.7865 & 9.2425 & 13.1797 & 15.8739 & 16.9136 \\
\hline & $M E$ & 0.3850 & 0.3106 & 0.2126 & 0.1969 & 0.1016 & 0.0778 \\
\hline \multirow[t]{7}{*}{ Fig. 4 (row2) } & $V_{P C}$ & 0.8337 & 0.4322 & 0.8119 & 0.7210 & 0.9445 & 0.9527 \\
\hline & $A c c$. & 0.9212 & 0.9092 & 0.7981 & 0.8949 & 0.9139 & 0.9306 \\
\hline & Sen. & 0.8818 & 0.8852 & 0.6972 & 0.8424 & 0.8709 & 0.8959 \\
\hline & Spe. & 0.9409 & 0.9212 & 0.8486 & 0.9212 & 0.9354 & 0.9479 \\
\hline & Pre. & 0.8818 & 0.8489 & 0.6972 & 0.8424 & 0.8709 & 0.8959 \\
\hline & $P S N R$ & 15.2652 & 12.9993 & 10.8876 & 13.9743 & 14.8771 & 15.8177 \\
\hline & $M E$ & 0.1182 & 0.1360 & 0.3027 & 0.1575 & 0.1290 & 0.1040 \\
\hline \multirow[t]{7}{*}{ Fig. 4 (row3) } & $V_{P C}$ & 0.8480 & 0.8532 & 0.8455 & 0.7445 & 0.9168 & 0.9237 \\
\hline & Acc. & 0.7798 & 0.8538 & 0.8678 & 0.9410 & 0.9342 & 0.9500 \\
\hline & Sen. & 0.6698 & 0.7941 & 0.8069 & 0.9115 & 0.9013 & 0.9562 \\
\hline & Spe. & 0.8349 & 0.8837 & 0.8475 & 0.9557 & 0.9506 & 0.9565 \\
\hline & Pre. & 0.6698 & 0.7735 & 0.7924 & 0.9115 & 0.9013 & 0.9481 \\
\hline & $P S N R$ & 10.2240 & 11.8814 & 12.2073 & 16.5526 & 15.9990 & 16.9005 \\
\hline & $M E$ & 0.3301 & 0.2191 & 0.2996 & 0.0884 & 0.0987 & 0.0923 \\
\hline
\end{tabular}

\subsubsection{Comparison of segmentation performance with Speckle noise}

Combine Fig. 7 and Table 6, the improved algorithm also works best when processing images contaminated by Speckle noise.

\subsubsection{Comparison of segmentation performance with Rician noise}

The improved algorithm also has the best segmentation effect for images polluted by Rician noise, see Fig. 8 and Table 7.
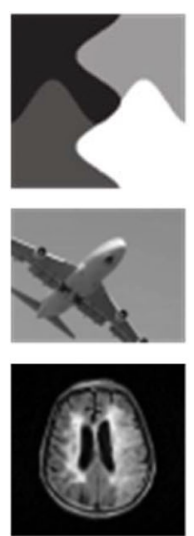

(a)
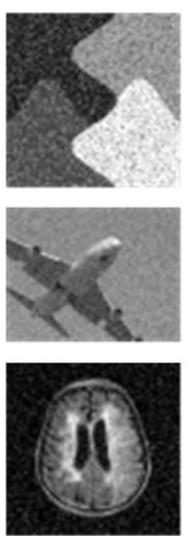

(b)
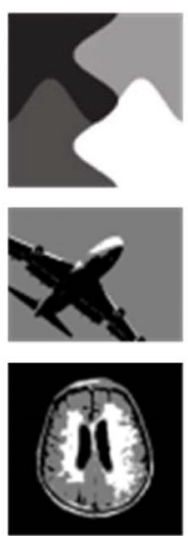

(c)
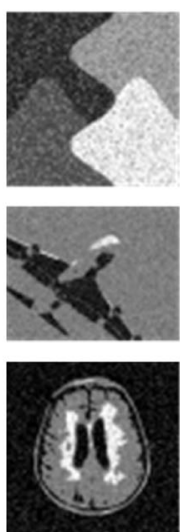

(d)
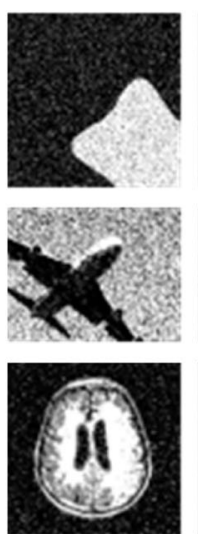

(e)
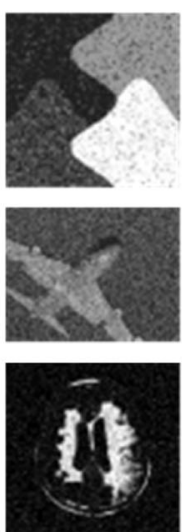

(f)
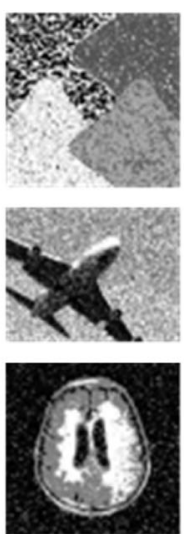

(g)
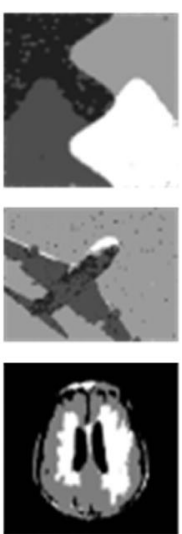

(h)
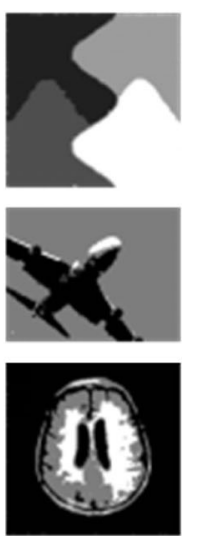

(i)

Fig. 5 The segmentation result of image polluted by 20\% Salt and Pepper noise a Original image b Noisy image c Ideal image d FCM result e BCFCM result f EnFCM result g FLICM result h FLICMLNLI result i Improved result 
Table 4 The performance index of different algorithms for segmenting original images polluted by Salt and Pepper noise

\begin{tabular}{|c|c|c|c|c|c|c|c|}
\hline Image & Index & FCM & BCFCM & EnFCM & FLICM & FLICMLNLI & Improved \\
\hline \multirow[t]{7}{*}{ Fig. 5 (row1) } & $V_{P C}$ & 0.9621 & 0.9662 & 0.9678 & 0.5949 & 0.9520 & 0.9712 \\
\hline & $A c c$. & 0.8022 & 0.7904 & 0.8049 & 0.7798 & 0.8131 & 0.8137 \\
\hline & Sen. & 0.8993 & 0.9021 & 0.9205 & 0.7283 & 0.9820 & 0.9868 \\
\hline & Spe. & 0.7954 & 0.7826 & 0.7675 & 0.7835 & 0.8012 & 0.8016 \\
\hline & Pre. & 0.2354 & 0.2252 & 0.2431 & 0.1907 & 0.2571 & 0.2583 \\
\hline & $P S N R$ & 12.1078 & 17.1673 & 18.1366 & 20.7153 & 23.6353 & 25.0068 \\
\hline & $M E$ & 0.1472 & 0.2010 & 0.2021 & 0.9034 & 0.0507 & 0.0209 \\
\hline \multirow[t]{7}{*}{ Fig. 5 (row2) } & $V_{P C}$ & 0.9487 & 0.4950 & 0.8971 & 0.5082 & 0.9258 & 0.9781 \\
\hline & $A c c$. & 0.7516 & 0.7373 & 0.8034 & 0.7418 & 0.7518 & 0.9811 \\
\hline & Sen. & 0.6908 & 0.7423 & 0.8084 & 0.6128 & 0.7147 & 0.9717 \\
\hline & Spe. & 0.7519 & 0.7323 & 0.7985 & 0.8064 & 0.7520 & 0.9858 \\
\hline & Pre. & 0.6119 & 0.7359 & 0.8004 & 0.6128 & 0.9227 & 0.9717 \\
\hline & $P S N R$ & 13.0782 & 7.4570 & 7.1626 & 9.9740 & 19.7807 & 21.4761 \\
\hline & $M E$ & 0.2843 & 0.3583 & 0.1921 & 0.3871 & 0.0324 & 0.0282 \\
\hline \multirow[t]{7}{*}{ Fig 5 (row3) } & $V_{P C}$ & 0.8579 & 0.7920 & 0.8572 & 0.9140 & 0.8953 & 0.9537 \\
\hline & Acc. & 0.9325 & 0.9415 & 0.9585 & 0.9595 & 0.9607 & 0.9774 \\
\hline & Sen. & 0.8987 & 0.9105 & 0.9377 & 0.9393 & 0.9411 & 0.9662 \\
\hline & Spe. & 0.9493 & 0.9720 & 0.9588 & 0.9696 & 0.9705 & 0.9831 \\
\hline & Pre. & 0.8987 & 0.9454 & 0.9377 & 0.9393 & 0.9411 & 0.9662 \\
\hline & $P S N R$ & 15.9910 & 16.8515 & 18.0636 & 18.2196 & 18.3524 & 20.7173 \\
\hline & $M E$ & 0.1012 & 0.0427 & 0.0622 & 0.0606 & 0.0588 & 0.0337 \\
\hline
\end{tabular}

\subsubsection{The influence of Guassian noise of different density on evaluation index}

Add Gaussian noise with different normalized variance values (from 0.15 to 1 ) to multiple pictures, get the partition coefficient and partition entropy of different algorithms,
Fig. 9a and $\mathrm{b}$ show the change of partition entropy and partition coefficient of each algorithm with the increase of gaussian noise intensity.

In addition, these algorithms are quantitatively compared by calculating the clustering indicators of the algorithms, as shown in the Fig. 10 for accuracy, sensitivity, and specificity.
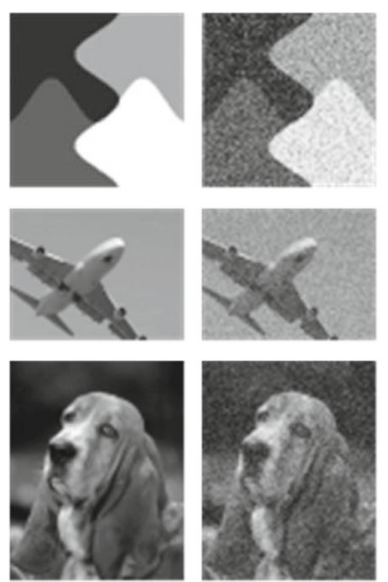

(a)

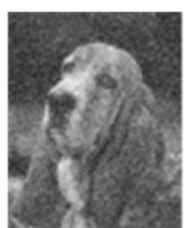

(b)

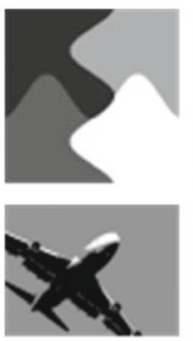

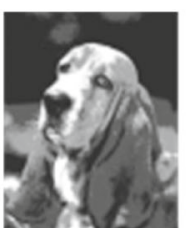

(c)
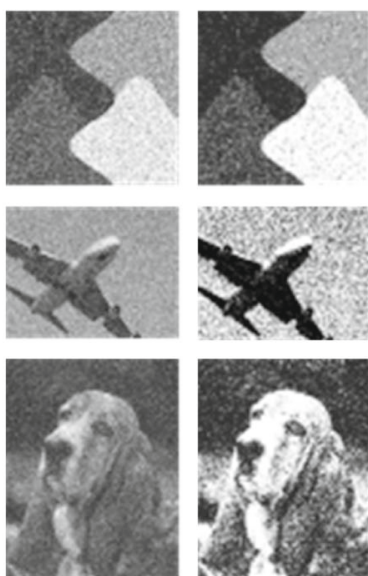

(d)

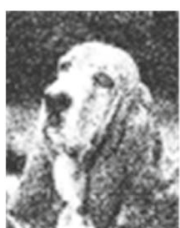

(e)
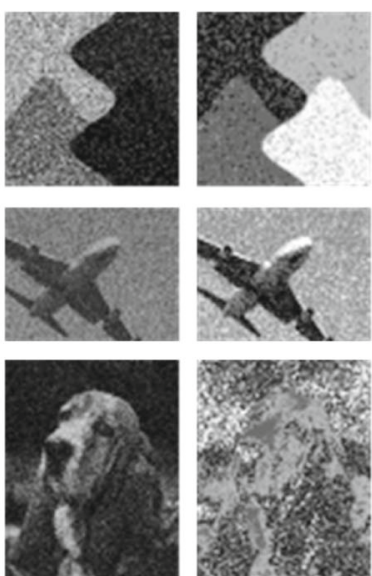

(f)

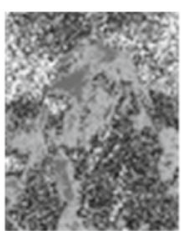

(g)
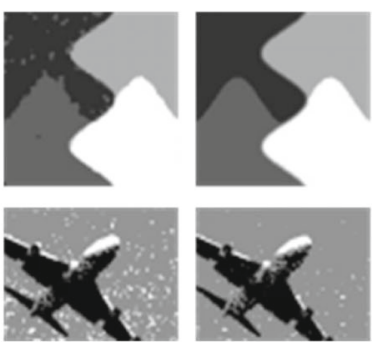

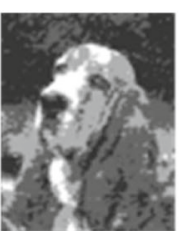

(h)

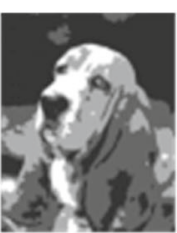

(i)

Fig. 6 The segmentation result of image polluted by Gaussian noise with a normalized variance of 0.05 and $10 \%$ Salt \& Pepper noise a Original image b Noisy image $\mathbf{c}$ Ideal image d FCM result e BCFCM result $\mathbf{f}$ EnFCM result $\mathbf{g}$ FLICM result h FLICMLNLI result i Improved result 
Table 5 The performance index of different algorithms for segmenting original images polluted by Mixed noise

\begin{tabular}{|c|c|c|c|c|c|c|c|}
\hline Image & Index & FCM & BCFCM & EnFCM & FLICM & FLICMLNLI & Improved \\
\hline \multirow[t]{7}{*}{ Fig. 6 (row1) } & $V_{P C}$ & 0.8169 & 0.7623 & 0.7765 & 0.8154 & 0.9582 & 0.9631 \\
\hline & Acc. & 0.7856 & 0.7941 & 0.7983 & 0.7897 & 0.80213 & 0.8123 \\
\hline & Sen. & 0.7725 & 0.9302 & 0.9162 & 0.8035 & 0.9633 & 0.9763 \\
\hline & Spe. & 0.7866 & 0.7846 & 0.7951 & 0.7887 & 0.7931 & 0.8008 \\
\hline & Pre. & 0.8022 & 0.8322 & 0.8787 & 0.9104 & 0.9435 & 0.9556 \\
\hline & $P S N R$ & 11.8608 & 17.0747 & 19.3139 & 20.2267 & 23.4971 & 23.7647 \\
\hline & $M E$ & 0.4251 & 0.2016 & 0.1933 & 0.0642 & 0.0513 & 0.0403 \\
\hline \multirow[t]{7}{*}{ Fig. 6 (row2) } & $V_{P C}$ & 0.7788 & 0.6187 & 0.6724 & 0.5249 & 0.9383 & 0.9594 \\
\hline & $A c c$. & 0.6829 & 0.4897 & 0.8421 & 0.8226 & 0.9425 & 0.9612 \\
\hline & Sen. & 0.5244 & 0.2446 & 0.7631 & 0.7340 & 0.9137 & 0.9418 \\
\hline & Spe. & 0.7622 & 0.6123 & 0.8815 & 0.8670 & 0.9568 & 0.9709 \\
\hline & Pre. & 0.5244 & 0.2398 & 0.7631 & 0.7340 & 0.9137 & 0.9418 \\
\hline & $P S N R$ & 8.6801 & 6.9533 & 12.2080 & 11.6838 & 16.5733 & 18.3942 \\
\hline & $M E$ & 0.4755 & 0.7652 & 0.2368 & 0.2659 & 0.0862 & 0.0581 \\
\hline \multirow[t]{7}{*}{ Fig. 6 (row3) } & $V_{P C}$ & 0.8337 & 0.4322 & 0.8119 & 0.7210 & 0.9445 & 0.9527 \\
\hline & Acc. & 0.8556 & 0.4481 & 0.8572 & 0.7655 & 0.9127 & 0.9396 \\
\hline & Sen. & 0.8862 & 0.7535 & 0.8826 & 0.9178 & 0.9731 & 0.9836 \\
\hline & Spe. & 0.8589 & 0.4982 & 0.8506 & 0.7481 & 0.9474 & 0.9683 \\
\hline & Pre. & 0.8171 & 0.4512 & 0.7758 & 0.6786 & 0.9433 & 0.9445 \\
\hline & $P S N R$ & 13.0782 & 17.4570 & 17.1626 & 19.9740 & 20.7807 & 22.4761 \\
\hline & $M E$ & 0.3843 & 0.2583 & 0.2921 & 0.2871 & 0.0519 & 0.0320 \\
\hline
\end{tabular}

\subsubsection{Further comparison with the current state-of-the-art algorithms}

By comparing the results of the algorithm in this paper with the classic algorithm in the previous sections, it is found that the optimized algorithm proposed in this paper has better segmentation results when segmenting images contaminated by different types of noise. In this section, we will select several superior algorithms recently published in journals with higher impact factors, including KWFLICM algorithm, APFCM algorithm, TFLICM algorithm, FALRCM algorithm and its fast algorithm [52-55], and compare them with the algorithms in this article.

In addition, a new indicator, Jacobian Score $(J S)$ [56] will be introduced to compare the performance of algorithms. The calculation formula of this indicator is as
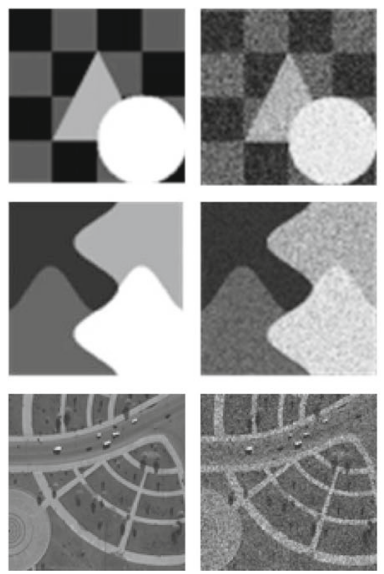

(a)

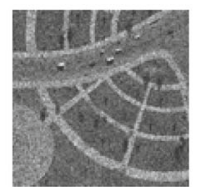

(b)
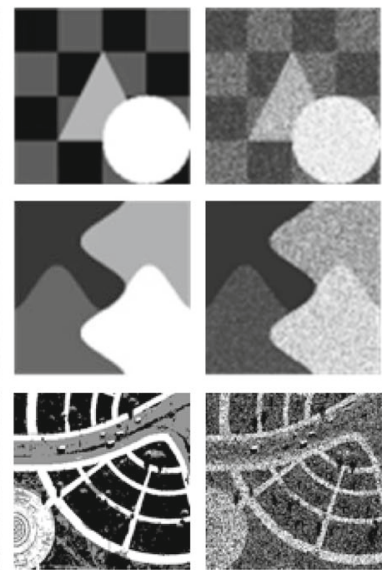

(c)

(d)
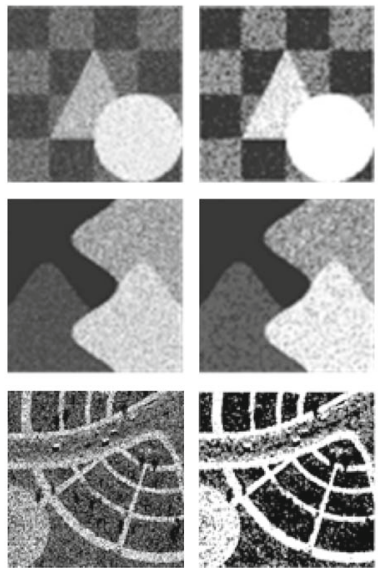

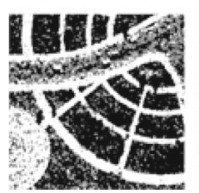

(e)
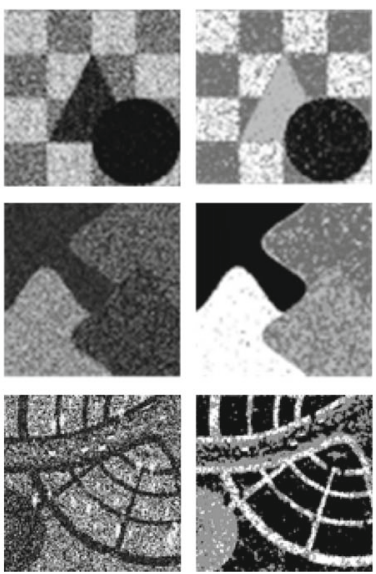

(f)

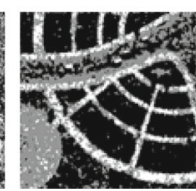

(g)
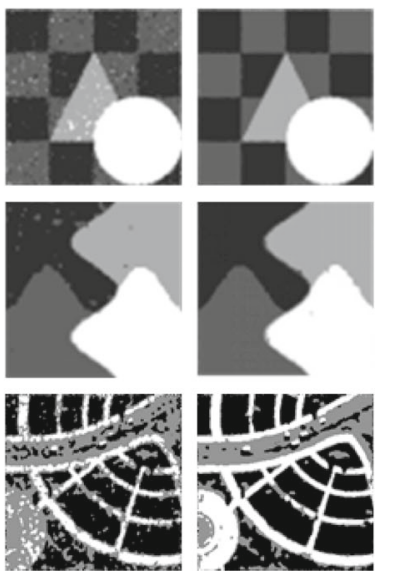

(h)

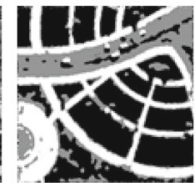

(i)

Fig. 7 The segmentation result of image polluted by Speckle noise with a normalized variance of 0.06 a Original image b Noisy image $\mathbf{c}$ Ideal image d FCM result e BCFCM result $\mathbf{f}$ EnFCM result $\mathbf{g}$ FLICM result $\mathbf{h}$ FLICMLNLI result i Improved result 
Table 6 The performance index of different algorithms for segmenting original images polluted by Speckle noise

\begin{tabular}{|c|c|c|c|c|c|c|c|}
\hline Image & Index & FCM & BCFCM & EnFCM & FLICM & FLICMLNLI & Improved \\
\hline \multirow[t]{7}{*}{ Fig. 7 (row1) } & $V_{P C}$ & 0.8708 & 0.6538 & 0.8627 & 0.8631 & 0.9523 & 0.9646 \\
\hline & Acc. & 0.9595 & 0.9489 & 0.9578 & 0.9482 & 0.9674 & 0.9694 \\
\hline & Sen. & 0.9393 & 0.9457 & 0.9124 & 0.9223 & 0.9512 & 0.9541 \\
\hline & Spe. & 0.9696 & 0.9352 & 0.9265 & 0.9612 & 0.9756 & 0.9770 \\
\hline & Pre. & 0.9393 & 0.9248 & 0.9404 & 0.9231 & 0.9512 & 0.9541 \\
\hline & $P S N R$ & 18.0853 & 18.7990 & 18.7482 & 17.2085 & 18.9820 & 19.3871 \\
\hline & $M E$ & 0.0606 & 0.0399 & 0.0585 & 0.0587 & 0.0487 & 0.0458 \\
\hline \multirow[t]{7}{*}{ Fig. 7 (row2) } & $V_{P C}$ & 0.8352 & 0.7539 & 0.8251 & 0.7535 & 0.9776 & 0.9818 \\
\hline & $A c c$. & 0.8372 & 0.8230 & 0.8337 & 0.7707 & 0.9618 & 0.9630 \\
\hline & Sen. & 0.8340 & 0.7827 & 0.8307 & 0.7784 & 0.9684 & 0.9689 \\
\hline & Spe. & 0.8152 & 0.4962 & 0.9676 & 0.5574 & 0.9600 & 0.9605 \\
\hline & Pre. & 0.8014 & 0.5635 & 0.8146 & 0.5745 & 0.9729 & 0.9787 \\
\hline & $P S N R$ & 11.2190 & 12.9125 & 15.1110 & 18.3549 & 19.4550 & 23.2931 \\
\hline & $M E$ & 0.4076 & 0.2450 & 0.2921 & 0.2871 & 0.0788 & 0.0360 \\
\hline \multirow[t]{7}{*}{ Fig. 7(row3) } & $V_{P C}$ & 0.7805 & 0.7250 & 0.7742 & 0.8606 & 0.8560 & 0.9405 \\
\hline & Acc. & 0.9231 & 0.6391 & 0.9421 & 0.9365 & 0.9481 & 0.9660 \\
\hline & Sen. & 0.9123 & 0.4743 & 0.9383 & 0.9048 & 0.9221 & 0.9891 \\
\hline & Spe. & 0.9432 & 0.7215 & 0.9454 & 0.9524 & 0.9610 & 0.9745 \\
\hline & Pre. & 0.9117 & 0.4600 & 0.8973 & 0.9048 & 0.9221 & 0.9391 \\
\hline & $P S N R$ & 16.1310 & 8.3194 & 16.3736 & 16.1687 & 16.9763 & 17.4656 \\
\hline & $M E$ & 0.0821 & 0.5390 & 0.5435 & 0.0951 & 0.0778 & 0.0308 \\
\hline
\end{tabular}

follows:

$J S=\frac{\left|P_{i} \cap Q_{i}\right|}{\left|P_{i} \cup Q_{i}\right|}$

Where $P_{i}$ denote the pixels belonging to the manual segmented image, $Q_{i}$ denote the pixels belonging to the experimental segmented image. The larger the $J S$, the better the effect of segmentation.

The segmentation results obtained by using different algorithms to segment different types of pictures under the influence of different noises are shown in Fig. 11, and the performance indicators are shown in Table 8. Among them, the first row of Fig. 11 shows the segmentation results of a medical image contaminated by Gaussian noise with a normalized variance of 0.1 , the second row of Fig. 11 shows the segmentation results of a medical images contaminated by $30 \%$ Salt and Pepper noise, the third row of Fig. 11 shows the segmentation result of a remote sensing images contaminated by Mixed noise(Gaussian noise with a normalized variance of 0.05 and $10 \%$ Salt \& Pepper noise),
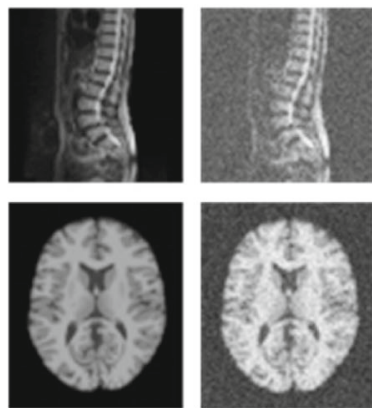

(a)
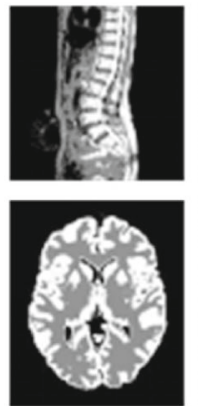

(c)
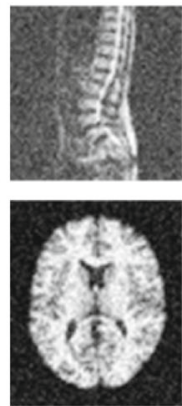

(d)
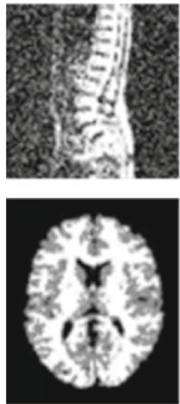

(e)
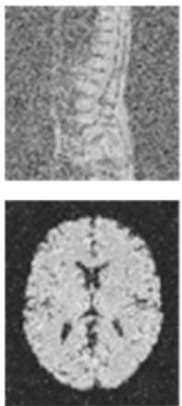

(f)
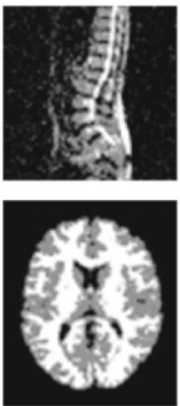

(g)
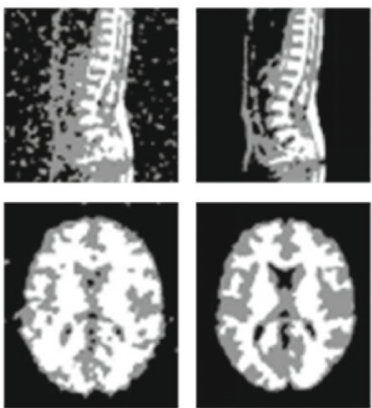

(h)

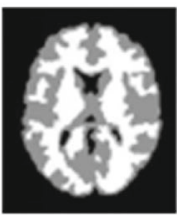

(i)

Fig. 8 The segmentation result of image polluted by Rician noise with standard deviation of 60 a Original image b Noisy image $\mathbf{c}$ Ideal image $\mathbf{d}$ FCM result e BCFCM result f EnFCM result g FLICM result h FLICMLNLI result i Improved result 
Table 7 The performance index of different algorithms for segmenting original images polluted by Rician noise

\begin{tabular}{|c|c|c|c|c|c|c|c|}
\hline Image & Index & FCM & BCFCM & EnFCM & FLICM & FLICMLNLI & Improved \\
\hline \multirow[t]{7}{*}{ Fig. 8 (row1) } & $V_{P C}$ & 0.8217 & 0.7047 & 0.7313 & 0.7677 & 0.9045 & 0.9249 \\
\hline & Acc. & 0.9176 & 0.7780 & 0.8581 & 0.8723 & 0.9294 & 0.9364 \\
\hline & Sen. & 0.8764 & 0.6808 & 0.7872 & 0.8085 & 0.8941 & 0.9046 \\
\hline & Spe. & 0.9382 & 0.8267 & 0.8936 & 0.9042 & 0.9470 & 0.9523 \\
\hline & Pre. & 0.8764 & 0.6626 & 0.7872 & 0.8085 & 0.8941 & 0.9046 \\
\hline & $P S N R$ & 15.1703 & 10.3265 & 12.7122 & 13.2301 & 15.7295 & 16.1575 \\
\hline & $M E$ & 0.1235 & 0.3322 & 0.2127 & 0.1914 & 0.1058 & 0.0953 \\
\hline \multirow[t]{7}{*}{ Fig. 8 (row2) } & $V_{P C}$ & 0.8273 & 0.7551 & 0.8812 & 0.9104 & 0.9731 & 0.9894 \\
\hline & $A c c$. & 0.8377 & 0.4221 & 0.8169 & 0.7213 & 0.9445 & 0.9755 \\
\hline & Sen. & 0.8554 & 0.4986 & 0.8531 & 0.7481 & 0.9474 & 0.9681 \\
\hline & Spe. & 0.8536 & 0.4453 & 0.8538 & 0.7633 & 0.9124 & 0.9389 \\
\hline & Pre. & 0.8569 & 0.6773 & 0.8639 & 0.9088 & 0.9740 & 0.9839 \\
\hline & PSNR & 9.24830 & 10.0515 & 11.3366 & 13.2891 & 18.4017 & 21.6958 \\
\hline & $M E$ & 0.3211 & 0.1830 & 0.1925 & 0.2725 & 0.0891 & 0.0448 \\
\hline
\end{tabular}

Fig. 9 The graph of the algorithm index changing with Gaussian normalized variance

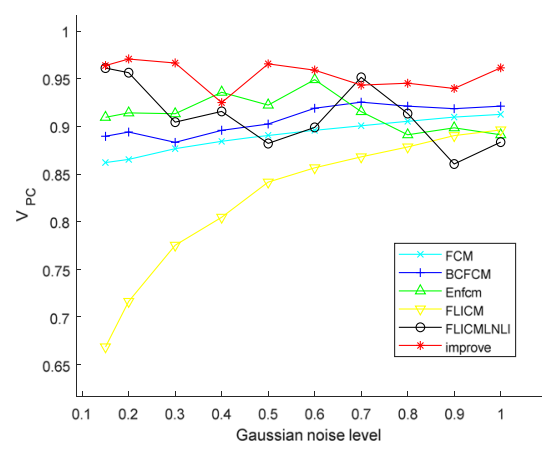

(a) Partition coefficient

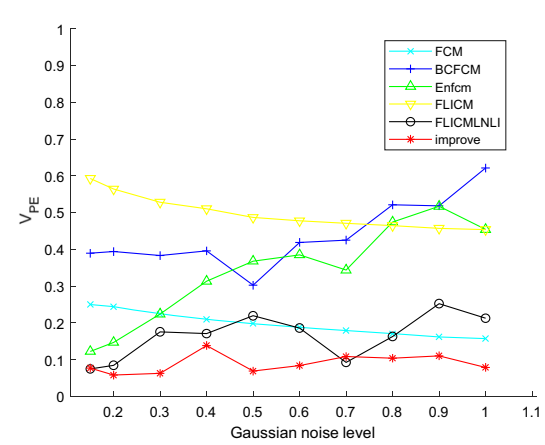

(b) Partition entropy

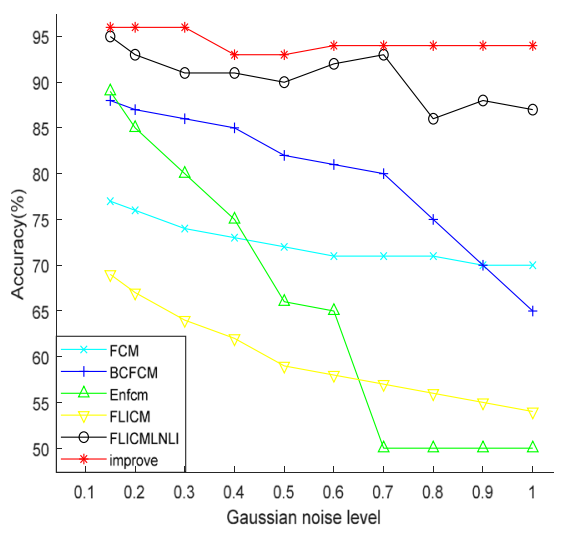

(a) Accuracy

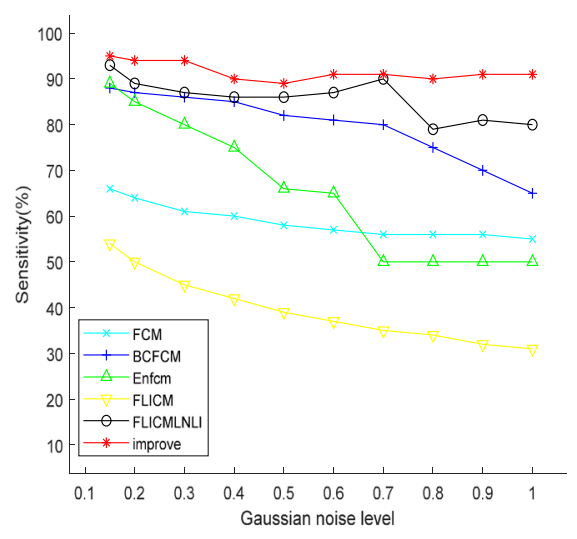

(b) Sensitivity

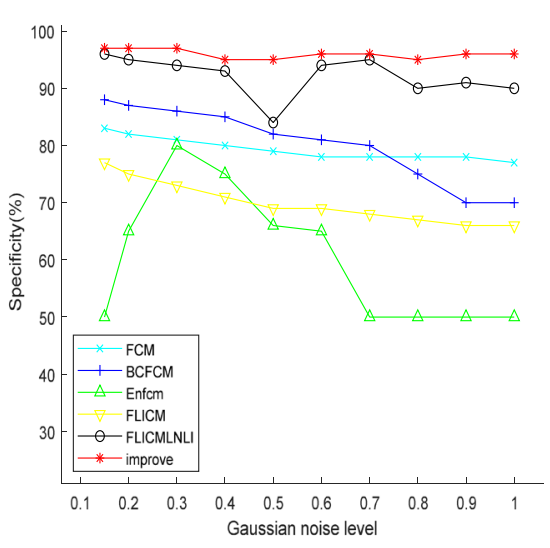

(c) Specificity

Fig. 10 Exponent and variance of Gaussian noise. a Accuracy; b Sensitivity; c Specificity 

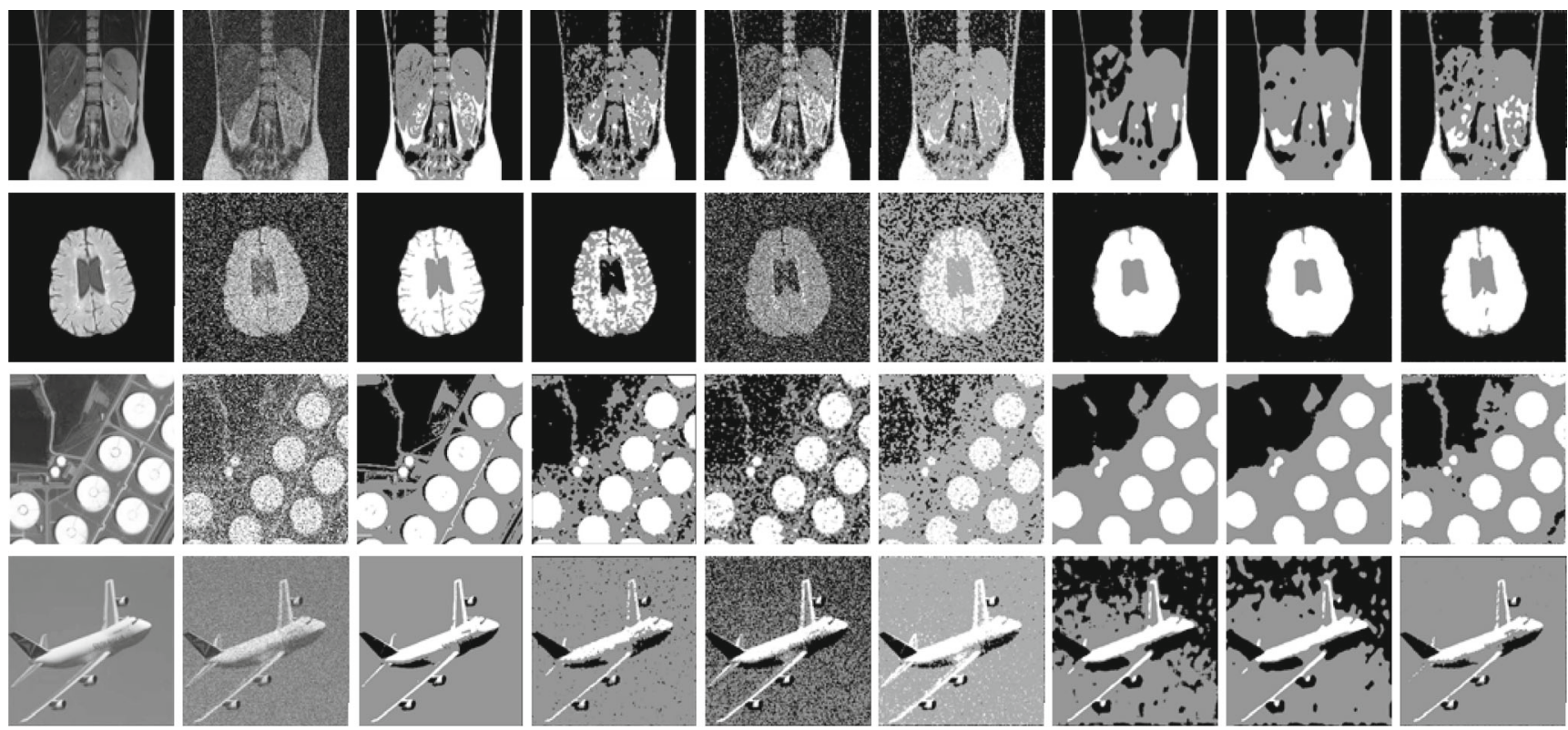

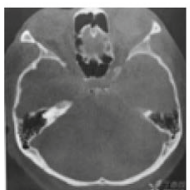

(a)

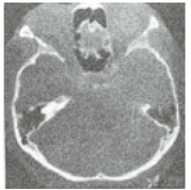

(b)

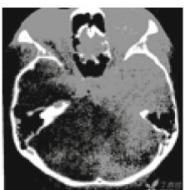

(c)

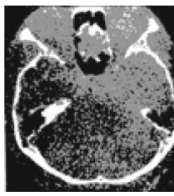

(d)

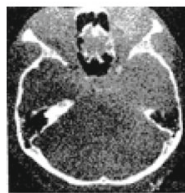

(e)

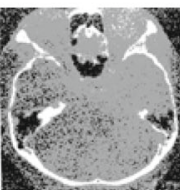

(f)

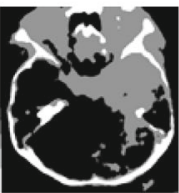

(g)

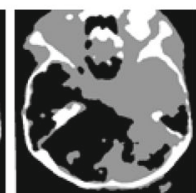

(h)

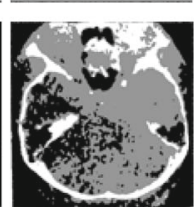

(i)

Fig. 11 Segmentation results of different algorithms a Original image b Noisy image $\mathbf{c}$ Ideal image $\mathbf{d}$ KWFLICM result e APFCM result $\mathbf{f}$ TFLICM result g FALRCM result h FALRCM_Fast result i Improved result

the fourth row of Fig. 11 shows the segmentation results of a natural image contaminated by Speckle noise with a normalized variance of 0.1 , The fifth row of Fig. 11 shows the segmentation results of a medical image contaminated by Rician noise with standard deviation of 60 .

From the comparison of segmented images and performance indicators shown above, it is not difficult to find that compared with the KWFLICM algorithm, APFCM algorithm and TFLCM algorithm, the algorithm proposed in this paper has better results when segmenting images contaminated by noise, and there are fewer noise points in the image. Compared with the FALRCM algorithm and its fast algorithm, which has more powerful anti-noise performance, the algorithm in this paper can retain more detailed image information when segmenting medical images with rich details.

\subsubsection{Complexity analysis and test of the improved algorithm}

Time complexity is also an important index to evaluate the performance of different algorithms. The higher the time complexity, the higher the calculation cost and the longer the execution time. The complexity of the algorithm proposed in this paper mainly comes from the introduction of non-local information in the new correlation model, the improved weighted Euclidian distance and the iterative process of the algorithm. For a grayscale image with $n$ pixels, the time complexity of correlation calculation according to the correlation model adopted in this paper is $O\left(n \times\left(R^{4}+R^{2}\right)\right)$. Where $R$ represents the size of the neighborhood window. For an image segmented into $c$ clusters, the time complexity of iterative calculation of the weighted distance $\sum_{i=1}^{n} \sum_{j=1}^{c} \omega_{1}\left(x_{i}-v_{j}\right)^{2}+\omega_{2}\left\|x_{i}^{N}-v_{j}^{N}\right\|^{2}$ is $O\left(l \times c \times n \times R^{2}\right)$, Where $l$ is the number of iterations. As a result, the total complexity of the proposed algorithm is $O\left(n \times\left(R^{4}+R^{2}\right)+l \times c \times n \times R^{2}\right)$. The time complexity comparison between the algorithm in this paper and some representative algorithms is shown in Table 9. The symbols in the table are as above.

It can be seen from Table 9 that the algorithm proposed in this paper is more complicated than other algorithms, so the time complexity is higher. The specific running time is compared as follows:

Firstly, the running time of the traditional algorithm and the improved algorithm is compared as shown in the Table 10. The running times of the first row in Fig. 5, the 
Table 8 Different algorithm segmentation indexes under different noises

\begin{tabular}{|c|c|c|c|c|c|c|c|}
\hline Image & Index & KWFLICM & APFCM & TFLICM & FALRCM & FALRCM_Fast & Improved \\
\hline \multirow[t]{5}{*}{ Fig. 11 (Guassian noise) } & $V_{P C}$ & 0.6229 & 0.6147 & 0.6851 & 0.9881 & 0.9984 & 0.9287 \\
\hline & Acc. & 0.9051 & 0.7865 & 0.6407 & 0.8359 & 0.8361 & 0.9498 \\
\hline & $P S N R$ & 12.7825 & 7.2987 & 7.5380 & 11.6362 & 11.6511 & 11.8300 \\
\hline & $M E$ & 0.1867 & 0.6798 & 0.5950 & 0.7538 & 0.7542 & 0.1773 \\
\hline & $J S$ & 0.8695 & 0.5331 & 0.3631 & 0.9659 & 0.9661 & 0.9818 \\
\hline \multirow[t]{5}{*}{ Fig. 11 (Salt and Pepper noise) } & $V_{P C}$ & 0.7993 & 0.6035 & 0.7062 & 0.9593 & 0.9685 & 0.9727 \\
\hline & Acc. & 0.6487 & 0.6861 & 0.78710 & 0.7621 & 0.8088 & 0.9319 \\
\hline & $P S N R$ & 7.1726 & 7.0158 & 9.0803 & 6.2650 & 6.3430 & 9.7587 \\
\hline & $M E$ & 0.6919 & 0.3722 & 0.6460 & 0.3243 & 0.3276 & 0.1235 \\
\hline & $J S$ & 0.8731 & 0.8403 & 0.8475 & 0.8853 & 0.8563 & 0.9705 \\
\hline \multirow[t]{5}{*}{ Fig. 11 (Mixed noise) } & $V_{P C}$ & 0.6270 & 0.6330 & 0.6386 & 0.9973 & 0.9985 & 0.9456 \\
\hline & Acc. & 0.8990 & 0.8401 & 0.8419 & 0.8933 & 0.8957 & 0.9226 \\
\hline & $P S N R$ & 13.9782 & 12.2295 & 11.3050 & 15.8438 & 15.9395 & 16.3333 \\
\hline & $M E$ & 0.1451 & 0.7602 & 0.6528 & 0.8933 & 0.8957 & 0.1160 \\
\hline & $J S$ & 0.8409 & 0.7499 & 0.7926 & 0.8874 & 0.8895 & 0.8969 \\
\hline \multirow[t]{5}{*}{ Fig. 11 (Speckle noise) } & $V_{P C}$ & 0.7106 & 0.6215 & 0.6520 & 0.6893 & 0.9729 & 0.9868 \\
\hline & Acc. & 0.9534 & 0.7514 & 0.9191 & 0.7585 & 0.7987 & 0.9623 \\
\hline & $P S N R$ & 18.9546 & 10.4639 & 15.3499 & 10.5727 & 11.3445 & 16.7323 \\
\hline & $M E$ & 0.0514 & 0.6271 & 0.8857 & 0.6378 & 0.6981 & 0.0328 \\
\hline & $J S$ & 0.9650 & 0.6223 & 0.9808 & 0.6311 & 0.6947 & 0.9896 \\
\hline \multirow[t]{5}{*}{ Fig. 11 (Rician noise) } & $V_{P C}$ & 0.6229 & 0.6147 & 0.6851 & 0.9881 & 0.9984 & 0.9287 \\
\hline & Acc. & 0.9051 & 0.7865 & 0.6407 & 0.8359 & 0.8361 & 0.9498 \\
\hline & $P S N R$ & 12.7825 & 7.2987 & 7.5380 & 11.6362 & 11.6511 & 11.8300 \\
\hline & $M E$ & 0.1867 & 0.6798 & 0.5950 & 0.7538 & 0.7542 & 0.1773 \\
\hline & $J S$ & 0.8695 & 0.5331 & 0.3631 & 0.9659 & 0.9661 & 0.9818 \\
\hline
\end{tabular}

first row in Fig. 6, and the first and second rows in Fig. 7 are averaged to get the first row in Table 10. The running times of three rows in Fig. 3, the second and third rows in Fig. 4, the third row in Fig. 5 and the two rows in Fig. 8 are averaged to get the second row in Table 10. Take the average running times of the first row in Fig. 4, the second row in Fig. 5, and the second and third rows in Fig. 6 to get the third row in Table 10. The last row in Table 10 shows the running time of the third row in Fig. 7.

Table 9 Time complexity comparison

\begin{tabular}{ll}
\hline Algorithm & Time complexity \\
\hline FLICM & $O(l \times c \times n \times R)$ \\
KWFLICM & $O(n \times R+l \times c \times n \times R)$ \\
APFCM & $O(c \times n+n \times R+l \times c \times n)$ \\
TFLICM & $O(l \times c \times n)$ \\
FLICMLNLI & $O\left(n \times\left(R^{4}+R^{2}\right)+l \times c \times n \times R\right)$ \\
Improved & $O\left(n \times\left(R^{4}+R^{2}\right)+l \times c \times n \times R^{2}\right)$ \\
\hline
\end{tabular}

Visualizing the data in Tables 10 and 11, the histogram of the running time of all algorithms is shown in Fig. 12.

From Tables 10, 11 and Fig. 12, it can be found that the improved algorithm proposed in this paper requires a relatively long running time. This is mainly due to the introduction of self-learning iterative methods and the simultaneous introduction of local and non-local information of the image. When segmenting different types of images, the execution efficiency of our algorithm is always lower than other comparison algorithms, especially the slowest speed when segmenting remote sensing images. This further shows that the real-time performance of the algorithm is poor. The improved algorithm can obtain better segmentation performance at the expense of real-time performance, which is one of the main deficiencies of the improved algorithm. However, this shortcoming will also provide new ideas for future research, and we will improve the running speed of this algorithm in the next research work.

Secondly, compare the running time of current state-ofthe-art algorithms and improved algorithm. The comparison 
Table 10 Compare the running time (in seconds) of traditional algorithms

\begin{tabular}{|c|c|c|c|c|c|c|}
\hline Image & FCM & BCFCM & EnFCM & FLICM & FLICMLNLI & Improved \\
\hline Synthetic images & 0.374 & 0.386 & 0.421 & 0.965 & 61.21 & 72.89 \\
\hline Medical images & 0.584 & 0.681 & 0.926 & 15.776 & 156.601 & 162.37 \\
\hline Natural images & 1.053 & 1.657 & 1.906 & 18.899 & 171.343 & 189.032 \\
\hline Remote sensing image & 0.181 & 0.338 & 0.677 & 0.805 & 191.84 & 219.098 \\
\hline
\end{tabular}

Table 11 Compare the running time (in seconds) of current state-of-the-art algorithms

\begin{tabular}{|c|c|c|c|c|c|c|}
\hline Image & KWFLICM & APFCM & TFLICM & FALRCM & FALRCM_Fast & Improved \\
\hline Medical images & 71.948 & 33.586 & 7.829 & 3.279 & 3.106 & 127.31 \\
\hline Natural image & 18.356 & 9.125 & 2.845 & 1.934 & 1.807 & 121.03 \\
\hline Remote sensing image & 4.109 & 2.844 & 3.907 & 1.396 & 1.384 & 190.43 \\
\hline
\end{tabular}

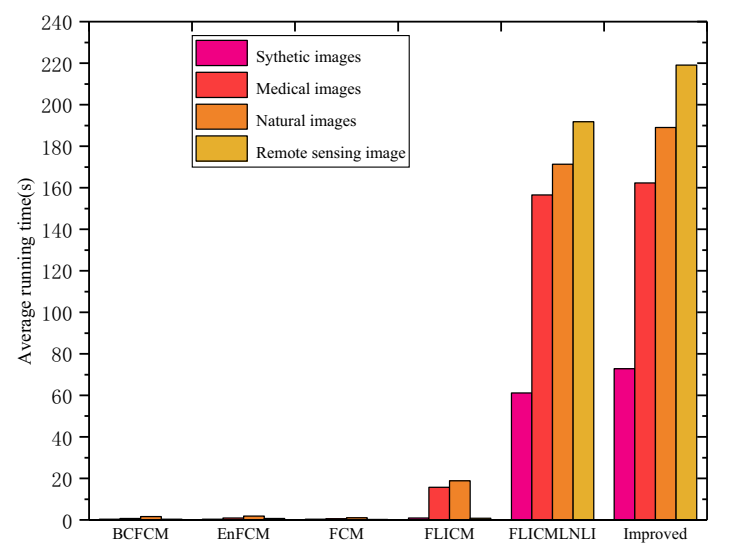

(a)

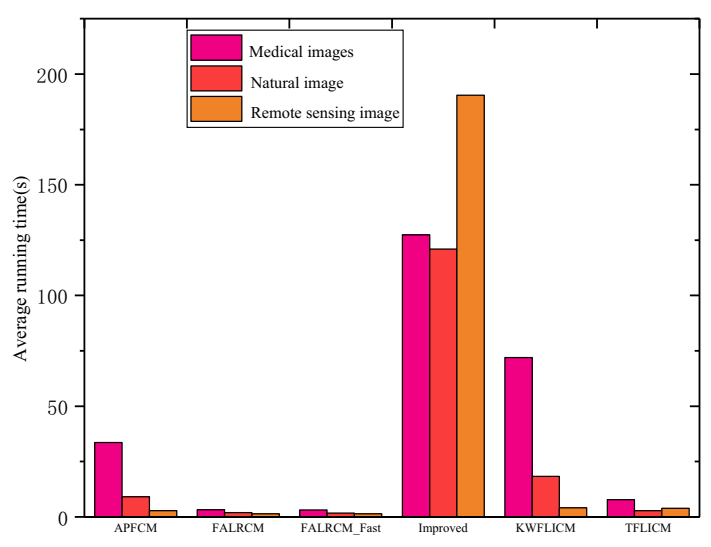

(b)

Fig. 12 Average running time (in seconds) on different types of images a Compare with traditional algorithms b Compare with current state-of-the-art algorithms 
results are shown in the Table 11 . The first row of data in Table 11 is obtained by calculating the average running time of the first, second, and fifth rows in Fig. 11, the second row of data in Table 11 is the running time of the fourth row in Fig. 11, the third row of data in Table 11 is the running time of the third row in Fig. 11.

\section{Conclusions}

Image segmentation has been an active area of research in the fields of computer vision and pattern recognition for the past two decades. In this paper, we propose a novel fuzzy algorithm, which can combine spatial information and iterative self-learning weighting into image segmentation. Based on the FLICMLNLI algorithm proposed by the predecessors, this paper believes that the Euclidean distance calculation formula that assigns the pixel information and its local information the same weight is unreasonable, so the determined different weight values are calculated through the iterative self-learning method. Pixel information and its local information have different weights, which is a more reasonable distance calculation method, and then it is embedded in the objective function to obtain an optimized model of image segmentation. This strategy make the proposed scheme capable of reducing heave noise and preserving more important details. After testing a large number of images with different types and numbers, it is found that no matter what kind of image is contaminated by noise, the improved algorithm is better than previous algorithms in terms of balancing noise removal ability and detail retention ability. Especially when processing detailed medical images, the improved algorithm can not only remove most of the noise, but also will not misprocess the detailed information such as veins and capillaries in the brain image. In general, the performance of the algorithm proposed in this paper is better.

Open Access This article is licensed under a Creative Commons Attribution 4.0 International License, which permits use, sharing, adaptation, distribution and reproduction in any medium or format, as long as you give appropriate credit to the original author(s) and the source, provide a link to the Creative Commons licence, and indicate if changes were made. The images or other third party material in this article are included in the article's Creative Commons licence, unless indicated otherwise in a credit line to the material. If material is not included in the article's Creative Commons licence and your intended use is not permitted by statutory regulation or exceeds the permitted use, you will need to obtain permission directly from the copyright holder. To view a copy of this licence, visit http://creativecommons. org/licenses/by/4.0/.

\section{References}

1. Mukul S, Nishi S (2013) The Origins of Digital Image Processing \& Application areas in Digital Image Processing Medical Images. Int J Eng Res Tech 1(2):48-52

2. Zaitoun NM, Aqel MJ (2015) Survey on image segmentation techniques. Procedia Comput Sci 65(4):797-806

3. Shivhare PK, Gupta V (2015) Review of Image Segmentation Techniques Including Pre \& Post Processing Operations[J/OL]. Int J Eng Adv Tech 4(3):153-157

4. Guo Q, Gao S, Zhang X, Yin Y, Zhang C (2018) Patch-Based Image Inpainting via Two-Stage Low Rank Approximation. IEEE Trans Vis Comput Graph 24(6):2023-2036

5. Jian M, Lam KM, Dong J, Shen L (2015) Visual-patch-attentionaware saliency detection. IEEE Trans Cybern 45(8):1575-1586

6. Liu Y, Cheng M, Hu X, Wang K, Bai X, Tang J (2019) Richer convolutional features for edge detection. IEEE Trans Pattern Anal Mach Intell 41(8):1939-1946

7. Liu H, Liu B, Zhang H, Li L, Qin X, Zhang G (2018) Crowd evacuation simulation approach based on navigation knowledge and two-layer control mechanism. Inf Sci 436-437:247-267

8. Liu H, Bin D, Zhang G (2018) A path planning approach for crowd evacuation in buildings based on improved artifificial bee colony algorithm. Appl Soft Comput 68:360-376

9. Sun Y, Jian M, Zhang X (2018) Reformed residual network with sparse feedbacks for $3 \mathrm{~d}$ reconstruction from a single image. IEEE Acc 6:70045-70052

10. Guo Z, Sun Y, Jian M, Zhang X (2018) Deep residual network with sparse feedback for image restoration. Appl Sci 8(12):1-10

11. Liu H, Xu J, Wu Y, Guo Q, Xing L (2018) Learning deconvolutional deep neural network for high resolution medical image reconstruction. Inf Sci 468:142-154

12. Lu W, Zhang X, Lu H, Li F (2020) Deep hierarchical encoding model for sentence semantic matching. J Visual Commun Image Represent:71

13. Otsu N (1979) A threshold selection method from gray-level histograms. IEEE Trans Syst Man Cybern 9(1):62-66

14. Park DC (2009) Classification of audio signals using fuzzy c-means with divergence-based kernel. Pattern Recognit Lett 30(9):794-798

15. Ruel JJ, Ayres MP (1999) Jensens inequality predicts effects of environmental variation. Trends Ecol Evol 14(9):361-366

16. Sharma PK, Holness G, Markushin Y, Melikechi N (2015) A family of Chisini mean based Jensen-Shannon divergence kernels. 14th IEEE Int. Conf. Mach. Learn. Appl., Miami, pp 109115

17. Shelhamer E, Long J, Darrell T (2017) Fully convolutional networks for semantic segmentation. IEEE Trans Pattern Anal Mach Intell 39(4):640-651

18. Ji J, Wang K L (2014) A Robust Nonlocal Fuzzy Clustering Algorithm with Between-Cluster Separation Measure for SAR Image Segmentation. IEEE J Sel Top Appl Earth Obser Remote Sens 7(12):4929-4936

19. Zhang H, Wang QM, Shi WZ et al (2017) A novel adaptive fuzzy local information $\mathrm{C}$-means clustering algorithm for remotely sensed imagery classification. IEEE Tran Geosci Remote Sens 55(9):5057-5068

20. Beevi SZ, Sathik MM, Senthamaraikannan K (2010) A Robust Fuzzy Clustering Technique with Spatial Neighborhood Information for Effective Medical Image Segmentation. Int J Comput Sci Inf Secur 7(3):1-8 
21. Sulaiman SN, Isa NAM (2010) Adaptive fuzzy-K-means Clustering Algorithm for Image Segmentation. IEEE Trans Consum Electron 56(4):2661-2668

22. Hong L, Jain A (1998) Integrating faces and fingerprints for personal identification. IEEE Trans Pattern Anal Mach Intell 20(12):295-1307

23. Chellappa R, Wilson C, Sirohey A (1995) Human and machine recognition of faces: a survey. Proc IEEE 83(5):705-741

24. Li QH, Ural S, Anderson J et al (2016) A fuzzy Mean-Shift approach to lidar waveform decomposition. IEEE Trans Geosci Remote Sens 54(12):7112-7121

25. Goyal S, Kumar S, Zaveri M et al (2017) Fuzzy similarity measure based spectral clustering framework for noisy image segmentation. Int J Uncertain Fuzz Knowl Based Syst 25(4):649_ 673

26. Chen K, Zou X, Xiong $J$ et al (2013) Improved fruit fuzzy clustering image segmentation algorithm based on visual saliency. Trans Chin Soc Agric Eng 29(6):157-165

27. Bezdek JC, Ehrlich R, Full WFCM (1984) The fuzzy c-means clustering algorithm. Comput Geosci 10(2):191-203

28. Bezdek JC, Hathaway RJ, Sabin MJ et al (1987) Convergence theory for fuzzy c-means: counterexamples and repairs. IEEE Trans Syst Man Cybern 17(5):873-877

29. Ahmed M, Yamany S, Mohamed N et al (2002) A modified Fuzzy C-Mean algorithm for bias field estimation and segmentation of MRI data. IEEE Trans Med Imaging 21(3):193-199

30. Chen SC, Zhang DQ (2004) Robust image segmentation using FCM with spatial constraints based on new kernel-induced distance measure. IEEE Trans Syst Man Cybern Part B Cybern 34(4):1907-1916

31. Szilagyi L, Benyo Z, Szilagyi SM et al (2003) MR Brain image segmentation using an enhanced fuzzy C-means algorithm. Proc 25th Ann Int Conf IEEE EMBS 1:724-726

32. Cai WL, Chen SC, Zhang DQ (2007) Fast and robust fuzzy cmeans clustering algorithms incorporating local information for image segmentation. Pattern Recognit 40(3):825-838

33. Krinidis S, Chatzis V (2010) A Robust Fuzzy Local Information C-Means Clustering Algorithm. IEEE Trans Image Process 19(5):1328-1337

34. Yu H, He F, Pan Y (2018) A novel region-based active contour model via local patch similarity measure for image segmentation. Multimed Tools Appl 77:24097-24119

35. Yu H, He F, Pan Y (2019) A novel segmentation model for medical images with intensity inhomogeneity based on adaptive perturbation. Multimed Tools Appl 78:11779-11798

36. Yu H, He F, Pan Y (2020) A scalable region-based level set method using adaptive bilateral filter for noisy image segmentation. Multimed Tools Appl 79:5743-5765

37. Zhang X, Sun Y, Liu H, Hou Z, Zhao F, Zhang C (2021) Improved Clustering Algorithms for Image Segmentation Based on Nonlocal Information and Back Projection. Inf Sci 550:129-144

38. Zhang X, Sun Y, Wang G et al (2017) Improved fuzzy clustering algorithm with non-local information for image segmentation. Multimed Tools Appl 76(6):1-27

39. Zhao J, Qiu X, Ma J et al (2016) Multi-objective optimization method of microgrid based on fuzzy clustering analysis and model recognition. Power Syst Technol 40(8):2316-2323
40. Zhao F, Li C, Liu H et al (2019) A multi-objective interval valued fuzzy clustering algorithm with spatial information for noisy image segmentation. J Intell Fuzzy Syst 36(6):5333-5344

41. Krinidis S, Krinidis M (2012) Generalised fuzzy local information C-means clustering algorithm. Electron Lett 48(23):1468-1470

42. Elazab A, Abdulazeem YM, Wu S et al (2016) Robust kernelized local information fuzzy C-means clustering for brain magnetic resonance image segmentation. J X-Ray Sci Technol 24(3):489507

43. Zhao F, Jiao L, Liu H (2013) Kernel generalized fuzzy c-means clustering with spatial information for image segmentation. Digit Signal Process 23(1):184-199

44. Xu R, Wunsch D (2005) Survey of clustering algorithms. IEEE Trans Neural Netw 16(3):645-678

45. Ahmed M, Yamany S, Farag A, Moriarty T (1999) Bias field estimation and adaptive segmentation of MRI data using a modified fuzzy C-means algorithm. IEEE Comput Soc 1:250-255

46. Tao X, Wang R, Chang R, Li C (2019) Density-sensitive fuzzy kernel maximum entropy clustering algorithm. Knowl Based Syst 166:42-57

47. Singh V, Verma NK (2019) An Entropy-based Variable Feature Weighted Fuzzy k-Means Algorithm for High Dimensional Data

48. Wu C, Yang XQ (2020) Robust credibilistic fuzzy local information clustering with spatial information constraints. Digital Signal Process 97:102615

49. Wu C, Wu QP (2017) A robust image segmentation algorithm based on the improved picture fuzzy clustering method on picture fuzzy sets. JXUPT 22(5):37-43

50. Gu YH, Sengur A (2012) A novel color image segmentation approach based on neutrosophic set and modified fuzzy C-means. Circ Syst Signal Process 32(4):1699-1723

51. Liu H, Xu J, Wu Y, Guo Q, Xing L (2018) Learning deconvolutional deep neural network for high resolution medical image reconstruction. Inf Sci 468:142-154

52. Gong M, Zhou Z, Ma J (2011) Change detection in synthetic aperture radar images based on image fusion and fuzzy clustering. IEEE Trans Image Process 1(4):2141-2151. https://doi.org/10.1109/TIP.2011.2170702

53. Wu C, Chen Y (2019) Adaptive entropy weighted picture fuzzy clustering algorithm with spatial information for image segmentation. Appl Soft Comput 86(105888). https://doi.org/10.1016/j.asoc.2019.105888

54. Wu C, Zhang X (2020) Total Bregman divergence-based fuzzy local information C-means clustering for robust image segmentation. Appl Soft Comput 94(106468). https://doi.org/10.1016/j.asoc.2020.106468

55. Wang Q, Wang K, Fang C, Jiao J (2021) Fuzzy image clustering incorporating local and region-level information with median memberships. Appl Soft Comput 105(107245). https://doi.org/10.1016/j.asoc.2021.107245

56. Vovk U, Pernus F, Likar B (2007) A review of methods for correction of intensity inhomogeneity in mri. IEEE Trans Med Imaging 26(3):405-421

Publisher's note Springer Nature remains neutral with regard to jurisdictional claims in published maps and institutional affiliations. 